\title{
Biochemical characterization and cloning of transglutaminases responsible for hemolymph clotting in Penaeus monodon and Marsupenaeus japonicus is
}

\author{
Maw-Sheng Yeh ${ }^{a}$, Ling-Rong Kao ${ }^{b}$, Chang-Jen Huang ${ }^{\text {b,c }}$, Inn-Ho Tsai ${ }^{\text {b,c,* }}$ \\ a Department of Food and Nutrition, Hung Kuang University, Sha Lu, Taiwan \\ ${ }^{\mathrm{b}}$ Institute of Biochemical Sciences, National Taiwan University, Taipei, Taiwan \\ ${ }^{\mathrm{c}}$ Institute of Biological Chemistry, Academia Sinica, Taipei, Taiwan
}

Received 29 October 2005; received in revised form 22 March 2006; accepted 7 April 2006

Available online 21 April 2006

\begin{abstract}
To investigate the shrimp blood clotting enzyme, a transglutaminase in the hemocytes of Penaeus monodon (abbreviated as TGH) was purified. TGH is an abundant homodimeric cytosolic protein with $84.2 \mathrm{kDa}$ subunits. It clotted shrimp plasma and incorporated fluorescent dansylcadaverine into succinyl casein upon activation by $\mathrm{CaCl}_{2}$ in vitro. $\mathrm{IC}_{50}$ for the activation was $3 \mathrm{mM}$, which is below the shrimp plasma Ca ${ }^{2+}$ level. Showing similar properties as other type II transglutaminase, TGH was particularly unstable after activation. MALDI-TOF/TOF mass-analyses of tryptic peptides of P. monodon TGH confirmed its identity to STG I (AY074924) previously cloned. A possible allele of the other isozyme STG II (AY771615) has also been cloned from the P. monodon cDNA and designated as PmTG. The predicted PmTG protein sequence is $58 \%$ similar to that of STG I and $99.2 \%$ to that of STG II. Likewise, a novel enzyme Mj-TGH was purified and cloned from Marsupenaeus japonicus hemocytes. Results of sequence alignment and phylogenetic analyses of these transglutaminases suggest that STG I and Mj-TGH are $83 \%$ identical and orthologous to each other, while PmTG/STG II and a previously cloned M.japonicus transglutaminase (AB162767) are their paralogs. Protein of the latter two could not be isolated, their regulated expression was discussed.
\end{abstract}

(C) 2006 Elsevier B.V. All rights reserved.

Keywords: Transglutaminase; Hemolymph coagulation; Regulation by $\mathrm{Ca}^{2+}$; cDNA sequence; Shrimp (Penaeus monodon and Marsupenaeus japonicus)

\section{Introduction}

Transglutaminases (EC 2.3.2.13) are known to play critical roles in blood coagulation and other biochemical process involving protein cross-linking or related super-structures. Enzymes in this family catalyze calcium-dependent acyl-transfer reactions between glutamine residues and lysine residues in protein substrates. The role of $\mathrm{Ca}^{2+}$ is to induce enzyme conformational changes essential for substrate-binding and catalysis $[1-4]$. The formation of intra-molecular or intermolecular $1-(\gamma-$

Abbreviations: Mj, Marsupenaeus japonicus; Pm, Penaeus monodon; TGH, hemocyte transglutaminase; RFE, relative fluorescence enhancement

it The cDNA sequences of PmTG and Mj-TGH have been deposited in GenBank with accession No. AF469484 and DQ436474, respectively.

* Corresponding author. Fax: +886223635038.

E-mail address: bc201@gate.sinica.edu.tw (I.-H. Tsai). glutamyl) lysine bonds lead to polymerized or insoluble proteins. In addition, the enzymes mediate other post-translational modification of proteins, e.g., deamidation and amine incorporation [1].

The enzymes are widely distributed in tissues and body fluids of animals. For example, blood coagulation factor XIIIa catalyzes the cross-linking of fibrin monomer during blood coagulation [5], another transglutaminase toughens skin epidermis and hair follicle epithelia [6] in vertebrates. The enzyme is also involved in forming the fertilization plug in female rodents [7]. Up to ten transglutaminases in various invertebrate species have so far been reported [8-11], including those responsible for blood coagulation in Crustacean $[9,10]$. The enzymes were also found to undergo acetylation [1], phosphorylation, or fattyacylation [12] that probably regulates their subcellular localization and catalytic activities. Some of them are involved in signal transduction and apoptosis $[1,13]$. 
Tiger shrimp (Penaeus monodon) is an economically important species cultured in Taiwan and southeastern Asia. Coagulation of the hemolymph is part of the innate immune response of Crustaceans, it prevents leakage of hemolymph from sites of injury and dissemination of invaders such as bacteria throughout the body. Much earlier, we have purified, characterized and cloned the clottable protein in the hemolymph of P. monodon [14]. Previously, two transglutaminases of P. monodon, STG I and STG II were cloned and expressed as the shrimp clotting enzymes $[8,10]$. In the present study, we first reported purification and biochemical characterization of major transglutaminases in shrimp hemocytes (abbreviated TGH) and their stability and regulation. Another transglutaminase from P. monodon (designated as PmTG), and a novel hemocyte transglutaminase designated as Mj-TGH from Marsupenaeus japonicus (kuruma prawn) were herein cloned and sequenced. Results of peptide sequencing and peptide mass fingerprinting of the purified TGHs from both shrimps enabled us to identify them with previously reported STG I of P. monodon [8] and Mj-TGH, respectively. Moreover, transglutaminases from invertebrates were subjected to phylogenetic analysis, and the regulations of their expression were discussed.

\section{Materials and methods}

\subsection{Shrimp hemocytes and the reagents}

A total of 90 live specimens of $P$. monodon and 50 live specimens of M. japonicus were obtained from local markets. The hemolymph was withdrawn by a syringe of $2.5 \mathrm{ml}$ with a needle of $23 \mathrm{G}$ and 1.25 inch long containing $1 / 8$ volume of anticoagulant $(0.1 \mathrm{M}$ sodium citrate, $0.4 \mathrm{M}$ $\mathrm{NaCl}$. It was centrifuged at $1600 \times \mathrm{g}$ for $5 \mathrm{~min}$ at $4{ }^{\circ} \mathrm{C}$, the supernatant was harvested for clotting assay. The pellet was washed twice with a cold buffer (1.5 mM EDTA, $0.4 \mathrm{M} \mathrm{NaCl}, 0.25 \mathrm{M}$ sucrose in $50 \mathrm{mM}$ Tris- $\mathrm{HCl}$ at $\mathrm{pH}$ 7.5). Casein and monodansylcadaverin were obtained from (Sigma Chem. Co., USA). Succinyl-casein was synthesized from casein and succinyl anhydride (Pierce, USA) as previously described [15].

\subsection{Transglutaminase assay}

Succinyl-casein $(500 \mu \mathrm{g} / \mathrm{ml})$ were mixed with $50 \mu \mathrm{M}$ monodansylcadaverine in $50 \mathrm{mM}$ Tris- $\mathrm{HCl}(\mathrm{pH}$ 7.6) containing 5-10 mM dithioerythreitol and $6 \mathrm{mM} \mathrm{CaCl}_{2}$. This substrate solution was thermostated at $23{ }^{\circ} \mathrm{C}$ in a cuvette before adding hemocyte lysate or purified P. monodon TGH Incorporation of dansylcadaverin to succinyl-casein by TGH was followed by a fluorospectrometer (model MPF-4, Hitachi, Japan) at the excitation/ emission wavelengths of $360 / 500 \mathrm{~nm}$, respectively [15]. Upon adding the $\mathrm{TGH}$, the reaction started immediately in the presence of $\mathrm{CaCl}_{2}$ and no lag phase was observed. Initial enzymatic rate within 1 min was measured to minimize errors due to enzyme instability, and relative fluorescence enhancement (RFE) was calculated based on the percent fluorescence increase relative to the blank. The shrimp plasma was diluted with equal volume of $50 \mathrm{mM}$ Tris $-\mathrm{HCl}(\mathrm{pH} 7.5)$ with $10 \mathrm{mM} \mathrm{CaCl}_{2}$ and then added purified TGH at a final concentration of $14-35 \mu \mathrm{g} / \mathrm{ml}$. The clotting time was monitored at $23{ }^{\circ} \mathrm{C}$ since the shrimps live in natural marine environment at $15-35{ }^{\circ} \mathrm{C}$.

\subsection{Purification and characterization of shrimp $T G H$}

After washing, the hemocytes in pellet were lysed by $10 \mathrm{mM}$ Tris-HCl buffer containing $1.5 \mathrm{mM}$ EDTA and $0.1 \mathrm{mM}$ ATP [16] and centrifuged to remove cell debris. The supernatant (i.e., hemocyte lysate) was loaded on a Fractogel TSK DEAE-650(M) column $(1.4 \times 7.5 \mathrm{~cm})$ at $4{ }^{\circ} \mathrm{C}$, which had been equilibrated in $50 \mathrm{mM}$ Tris- $\mathrm{HCl}$ buffer (pH 7.5) with $1.5 \mathrm{mM}$ EDTA and $0.1 \mathrm{mM}$ ATP. The sample was eluted with a linear gradient of $0-0.3 \mathrm{M} \mathrm{NaCl}$, fractions of $1 \mathrm{ml}$ were collected and assayed for transglutaminase activity. Active fractions were pooled and further purified by a Sephacryl S-300 column $(0.9 \times 60 \mathrm{~cm})$ at $4{ }^{\circ} \mathrm{C}$, which was equilibrated and eluted with the $50 \mathrm{mM}$ Tris $-\mathrm{HCl}$ buffer containing $1.5 \mathrm{mM}$ EDTA and $0.1 \mathrm{mM}$ ATP.

Molecular weight and purity of $P$. monodon TGH were studied by SDSPAGE [17]. Native molecular weight of TGH was also estimated by elution volume on a gel filtration column, by interpolation from a calibration plot of "ratios of the elution volume to the void volume" against log molecular weight of the markers. In addition, matrix-assisted laser desorption/ionization-time-offlight (MALDI-TOF) mass spectrometer (Model G2025, Hewlett-Packard, USA) was used to determine its accurate mass, in which $1 \mu 1$ of purified TGH $(0.4 \mathrm{mg} / \mathrm{ml})$ after dialysis against distilled water was added with $1 \mu 1$ sinapinic acid before crystallized under vacuum.

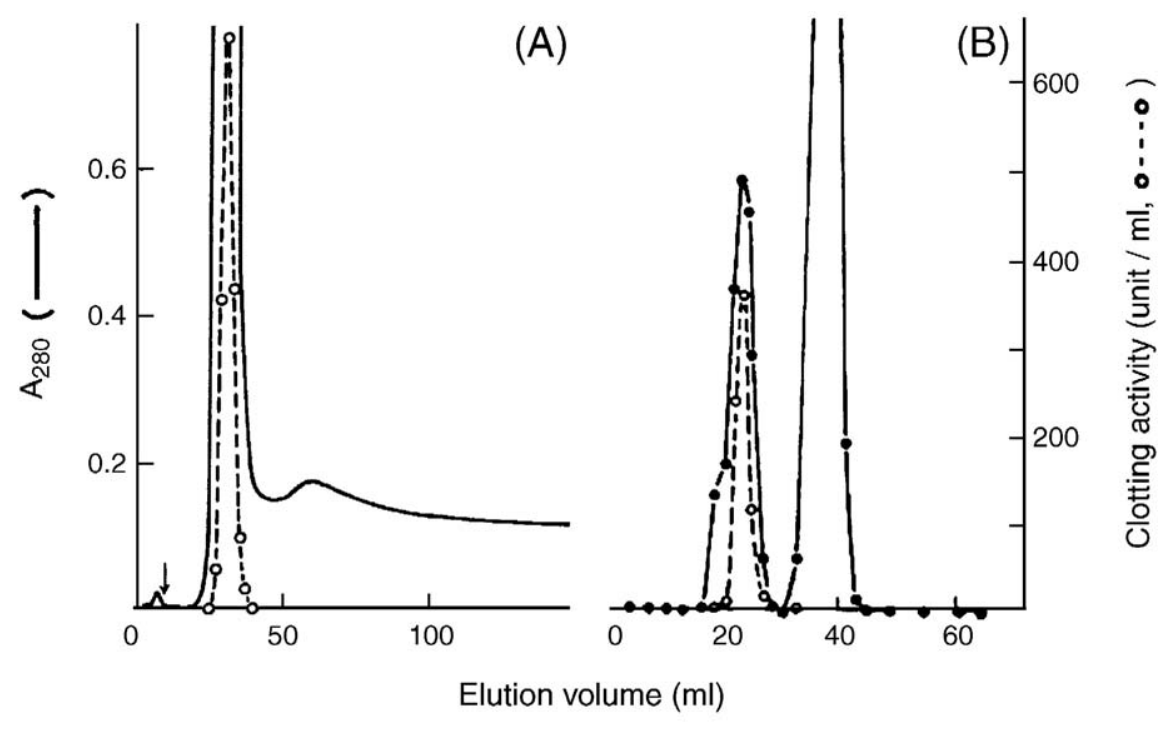

Fig. 1. Purification of TGH from P. monodon hemocyte lysate. (A) About $12 \mathrm{ml}$ hemocyte lysate was separated by a Fractogel TSK DEAE-650 (M) column at $4{ }^{\circ} \mathrm{C}$. The column was eluted with a gradient of $0-0.3 \mathrm{M} \mathrm{NaCl}$ in $50 \mathrm{mM}$ Tris $-\mathrm{HCl}(\mathrm{pH} 7.5)$, containing $1.5 \mathrm{mM}$ EDTA and $0.1 \mathrm{mM}$ ATP. Arrow indicated start of the gradient. (B) partially purified TG was subjected to gel filtration on a Sephacryl S-300 column. Fractions of $1 \mathrm{ml}$ were collected at $4{ }^{\circ} \mathrm{C}$, and transglutaminase activity $(\mathrm{O}-\mathrm{O})$ was assayed as detailed in Materials and methods. 
(A)

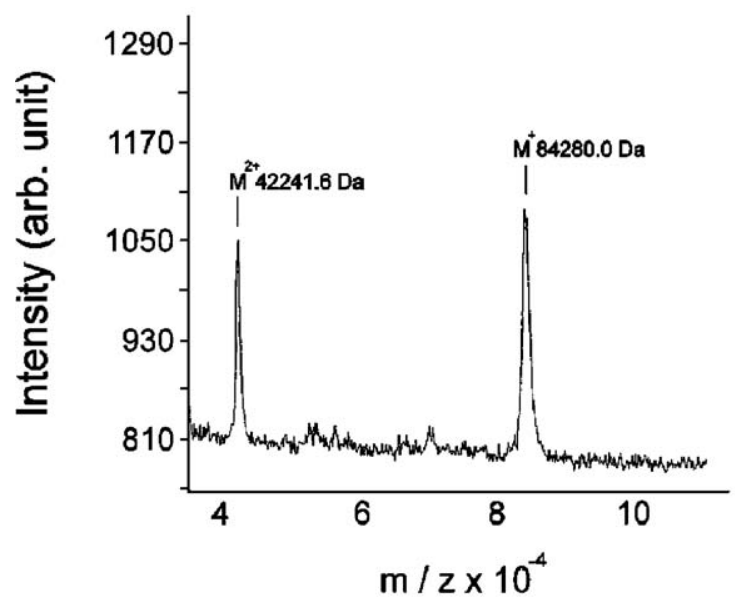

(B)
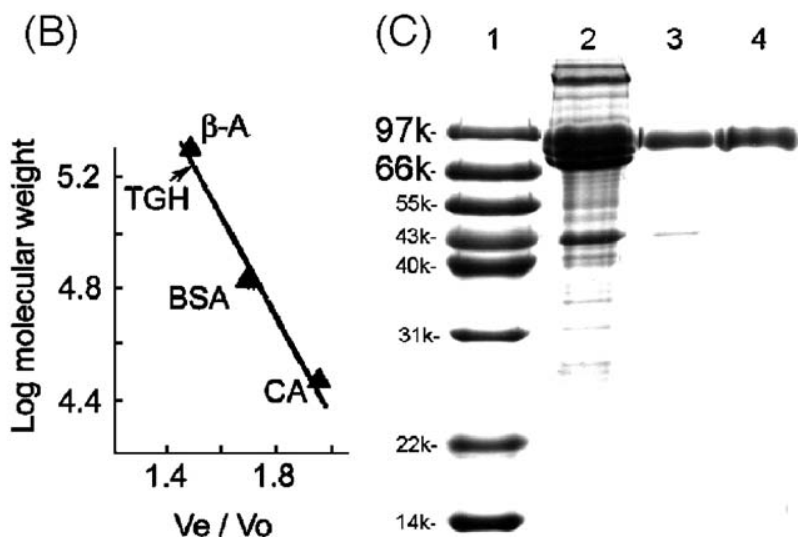

Fig. 2. (A) Molecular mass of purified P. monodon TGH as determined by MALDI-TOF mass spectrometry. (B) Estimation of the TGH molecular weight by gel filtration. Protein markers used were: $\beta$-amylase $(\beta-A, 200 \mathrm{kDa})$, bovine serum albumin (BSA, $67 \mathrm{kDa}$ ) and carbonic anhydrase (CA, $29 \mathrm{kDa}) . V_{\mathrm{e}}$ and $V_{\mathrm{o}}$ are elution-volumes for the protein marker and blue-dextran, respectively. (C) SDS-PAGE on $12 \%$ acrylamide gel. Lane 1, molecular weight markers; lane 2, hemocyte lysate; lanes 3 and 4 purified TGH with non-reducing and reducing sample buffer, respectively. Molecular weights of the markers were shown at left.

\subsection{Protein fragmentation and amino acid sequencing}

Likewise, the enzyme Mj-TGH was purified from the hemocytes of another aqua-cultured species $M$. japonicus. Both TGHs $(1 \mathrm{mg})$ of $P$. monodon and $M$. japonicus were denatured in $100 \mu \mathrm{l}$ of $6 \mathrm{M}$ guanidine hydrochloride in $0.2 \mathrm{M}$ Tris- $\mathrm{HCl}(\mathrm{pH} 8.5)$ and reduced by dithiothreitol at $50{ }^{\circ} \mathrm{C}$ for $1 \mathrm{~h}$. After adding excess iodoacetic acid and incubated at room temperature for $30 \mathrm{~min}$, the Scarboxymethyl protein was desalted and lyophilized. Amino acid sequences were analyzed by an automatic sequencer (Model 477A, Applied Biosystem, USA).

For fragmentation, M. japonicus TGH was dissolved in $100 \mu 1$ of $70 \%$ formic acid with $0.2 \mathrm{mg}$ of $\mathrm{CNBr}$ at room temperature for $24 \mathrm{~h}$. In addition, it was digested with Lys-C endopeptidase (Promega, Madison, WI, USA) in $50 \mathrm{mM}$ Tris- $\mathrm{HCl}(\mathrm{pH} 8.0)$ at $37^{\circ} \mathrm{C}$ for $20 \mathrm{~h}$ at an enzyme to substrate ratio of 1:50 (w/w). The reaction was stopped by adding dithiothreitol and heated at $95^{\circ} \mathrm{C}$ for $5 \mathrm{~min}$. Then the oligopeptides were purified by reversed-phase HPLC on a $\mathrm{C}_{18}$ column, then $\mathrm{N}$-terminal sequences of three of the fragments.

\subsection{Enzyme assay and effect of calcium and other reagents}

For the fluorescence assay, $\mathrm{CaCl}_{2}$ at a final concentration of $10 \mathrm{mM}$ was added to $P$. monodon TGH $(77 \mu \mathrm{g} / \mathrm{ml})$ in $50 \mathrm{mM}$ Tris- $\mathrm{HCl}, 1.5 \mathrm{mM}$ EDTA, $0.1 \mathrm{mM}$ ATP $(\mathrm{pH} 7.5)$ to start the reaction and initial rate $(\mathrm{RFL} / \mathrm{min})$ was measured [15]. For clotting assay, clotting time was measured after adding the enzyme to a buffer containing $60 \mu 1$ supernatant of the centrifuged hemolymph and equal volume of $50 \mathrm{mM}$ Tris- $\mathrm{HCl}(\mathrm{pH} 7.5)$ containing $20 \mathrm{mM} \mathrm{CaCl}_{2}$.

To investigate its thermostability, purified TGH in the buffer $(32 \mu \mathrm{g} / \mathrm{ml})$ were incubated at various temperature up to $50{ }^{\circ} \mathrm{C}$. After a period of time, $30 \mu 1$ aliquots were withdrawn for the fluorescence assay. In addition, we have studied effects of pre-incubation of TGH with the chlorides of IIA group metal ion or iodoacetamide which modified active site $\mathrm{CysSH}$ of TGH. The remaining enzyme activity was assayed by the fluorescence assay.

\section{6. $c D N A$ synthesis and cloning}

Standard procedures in molecular biology were used for preparation of plasmid DNA, restriction enzyme digestion, DNA agarose gel electrophoresis, DNA ligation, and the transformation of bacteria [18]. Total RNA was purified from tiger shrimp and kuruma prawn using the RNAzol B kit (Biotecx, Friendswood, TX, USA) [19]. The mRNA was purified using QuickPrepR Micro mRNA purification kit (Amersham Pharmacia, Freiburg, Germany). The first strand cDNA was synthesis using oligo(dT)-primer and random primers, and it was used as templates in subsequent polymerase chain reaction (PCR) [20].

To amplify the transglutaminase cDNAs of both species, we have used an antisense oligo(dT) and another degenerate primer designed from the conserved protein sequence (VPAHTIK) of M. japonicus TGH, i.e., sense 5'-GTNCCN

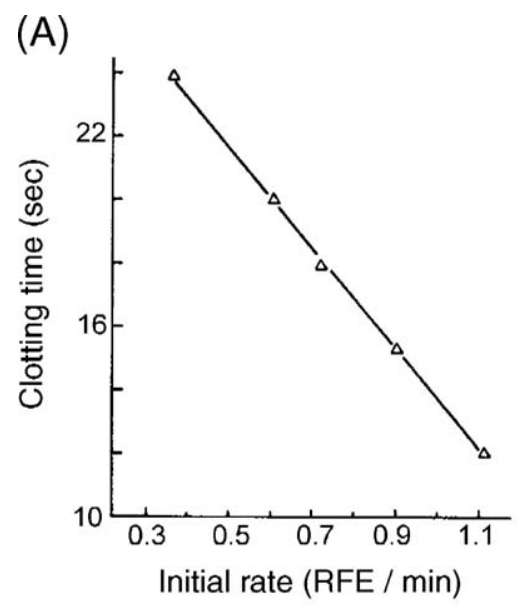

(B)

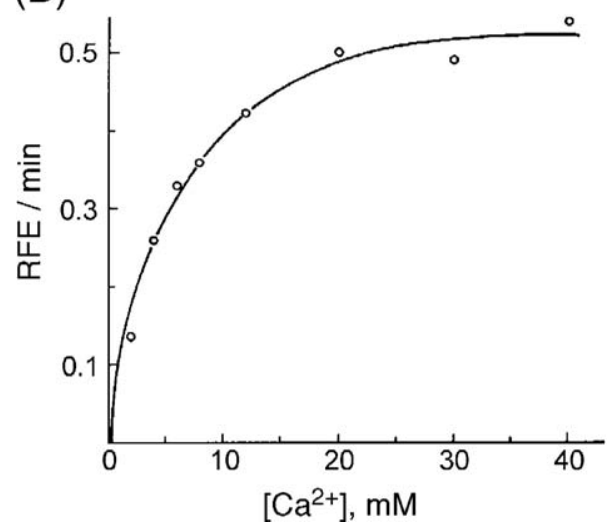

Fig. 3. (A) Correlation between clotting and amine-incorporating activities of purified TGH. After adding Tris- $\mathrm{HCl}$ buffer containing $\mathrm{CaCl}_{2}$ to the same volume of shrimp plasma, the clotting was started from the addition of TGH (final TGH concentration ranged from 14 to $35 \mu \mathrm{g} / \mathrm{ml}$ ). In separate experiments, initial rates of dansylcadaverin incorporation were determined by fluorescence assay. (B) Apparent $\mathrm{Ca}^{2+}$ dependency of $P$. monodon TGH activity. Purified TGH $(18 \mu \mathrm{g} / \mathrm{ml})$ was used in the fluorescence assay at various $\mathrm{CaCl}_{2}$ concentrations in Tris buffer at $23^{\circ} \mathrm{C}$. 


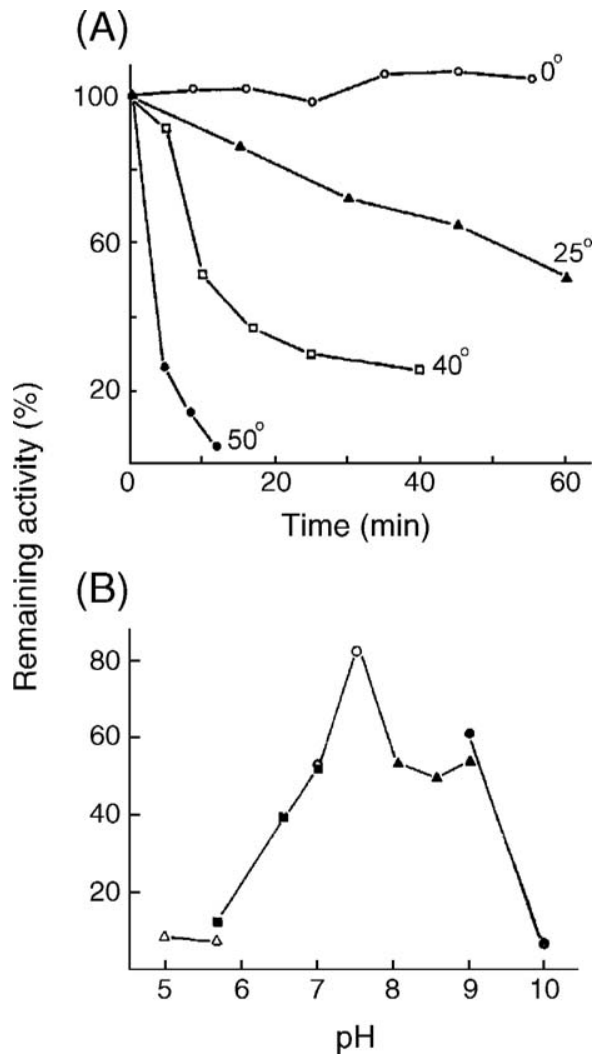

Fig. 4. (A) Thermostability of P. monodon TGH. The enzyme $(0.3 \mathrm{mg}$ protein $/ \mathrm{ml})$ in $50 \mathrm{mM}$ Tris- $\mathrm{HCl}, 1.5 \mathrm{mM}$ EDTA ( $\mathrm{pH} 7.5$ ) was pre-incubated at different temperature $\left(0-50^{\circ} \mathrm{C}\right)$ for $10 \mathrm{~min}$. At indicated time, aliquots were withdrawn for amine-incorporation assay. (B) Stability of $P$. monodon TGH at various $\mathrm{pH}$. The hemocyte lysate was diluted with $0.1 \mathrm{M}$ of the following buffers with $0.4 \mathrm{mM}$ EDTA at $4{ }^{\circ} \mathrm{C}$ and incubated $16 \mathrm{~h}$ before the amine-incorporation assay at $23^{\circ} \mathrm{C}$; sodium acetate $(\mathrm{pH} 5.0-5.8)(\triangle-\triangle)$, imidazole- $\mathrm{HCl}(\mathrm{pH} 5.8-7.0)(\mathbf{\square}-\mathbf{\square})$,

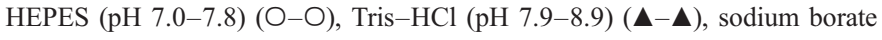
$(\mathrm{pH} 9.0-10)(\cdot-\cdot)$.

CANCA(TC)ACNAT(TCA) AA- $3^{\prime}$. Except for the first and last cycles, each of the 40 cycles was: $94{ }^{\circ} \mathrm{C}$ for $30 \mathrm{~s}, 42^{\circ} \mathrm{C}$ for $30 \mathrm{~s}$, and $72{ }^{\circ} \mathrm{C}$ for $1 \mathrm{~min}$. The first cycle included an extended denaturation time $(2 \mathrm{~min})$ during which polymerase was added while the last cycle had an extended (10 min) elongation period. The cDNAs of $578 \mathrm{bp}$ and $1071 \mathrm{bp}$ were thus produced from tiger shrimp and kuruma prawn, respectively. These cDNA were purified and ligated into pGEM-T (Promega). Each clone was sequenced by using AmpliTaq DNA polymerase and fluorescent dideoxynucleotides on a DNA sequencer (Applied Biosystems model 310) [21].

\subsection{Rapid amplification of $c D N A$ ends (RACE)}

Total RNA was isolated from both shrimps and poly (A) ${ }^{+}$RNA was prepared. The first-strand cDNA was synthesized using a modified poly-T primer and $1 \mu \mathrm{g}$ of poly $(\mathrm{A})^{+}$RNA. The second-strand cDNA was obtained using an enzyme cocktail containing RNase H, DNA polymerase, and DNA ligase. Asymmetric adaptor primers (AP primers) were then ligated to both ends of the double-stranded cDNA. An aliquot of this cDNA was diluted 1:100 and subjected to PCR. The 5' ends of mRNAs of PmTG and Mj-TGH were obtained by RACE methods using the Marathon cDNA amplification kit (Clontech, Palo Alto, CA, USA). Adaptor primers AP1 (external) and AP2 (internal) were supplied with the kit. RACE was performed with a 27 mer sense primer (AP1) and antisense primers, 5'-GTAGTAGTCACCGTACGTCACTTCC-3' for PmTG/ TGH and 5'-TGATAAGGTCATGGTCCACCAGCTTG-3' for $\mathrm{Mj-}$ TGH, respectively. The second round of PCR was carried out with a nested 25 mer sense primer (AP2) and nested antisense primers, $5^{\prime}$-CTTCGAGAAC GATTTCTCCCTCACG-3' for TGH and 5'-GTAGTAGTCAGAGTAGTA
CACCTCC- $3^{\prime}$ for Mj-TGH, respectively. Except for the first and last cycles, each cycle was: $94{ }^{\circ} \mathrm{C}$ for $30 \mathrm{~s}, 63{ }^{\circ} \mathrm{C}$ for $30 \mathrm{~s}$, and $72{ }^{\circ} \mathrm{C}$ for $1.5 \mathrm{~min}$. The first cycle included an extended denaturation time $(2 \mathrm{~min})$ during which polymerase was added while the last cycle had an extended $(10 \mathrm{~min})$ elongation period. After 40 PCR cycles, the products were cloned into pGEM-T vector (Promega) and sequenced $[20,21]$.

\subsection{RNA isolation from P. monodon tissues and RT-PCR}

Total RNA was isolated from gill, heart, hemocyte, hepatopancreas, intestine, lymphoid organ, and muscle of $P$. monodon using the RNAzol reagent (Biotecx, Friendswood, TX, USA). RNAase-free DNase (Promega) was added to eradicate the genomic DNA contamination. First-strand cDNA was amplified from $2 \mu \mathrm{g}$ total RNA using 10 pmole oligo-dT primer in a $25-\mu 1$ reaction containing $30 \mathrm{U}$ RNasin (Promega), $1 \mathrm{mM}$ dNTP, $10 \mathrm{mM}$ dithiothreitol, and $300 \mathrm{U}$ Superscript II (Life Technologies). After being incubated at $42{ }^{\circ} \mathrm{C}$ for $1 \mathrm{~h}$ in $1.5 \mathrm{mM} \mathrm{MgCl}_{2}, 0.25 \mathrm{mM} \mathrm{dNTP}$ and $0.5 \mathrm{U}$ ExTaq (Takara Shuzo Co., Japan), $2 \mu 1$ of the solution were added to $48 \mu 1$ solution of the primers (designed from PmTG; forward, 5'-TGGAAGTGACGTACGGTGAC TACTAC G-3' and reverse, 5'-GTTGTACAGCTGGCTGGAGTTGAAGG-3'). After amplified by PCR at the annealing temperature of $60{ }^{\circ} \mathrm{C}$ for $30 \mathrm{~s}$ ( $25-40$ cycles), the $390 \mathrm{bp}$ PCR products were examined on a $1.2 \%$ agarose gel.

\subsection{Molecular phylogenetic analysis}

Sequences of invertebrate transglutaminases were retrieved by BlastP [22]. Their amino acid sequences were aligned with the program CUSTAL W [23]. Phylogenetic tree was build by the program PHYLIP (http://www. evolution. genetics.washington.edu./phylip.html) [24]. Degree of confidence of the lineage at each node was determined by the bootstrap analyses of 1,000 replicates [25].

\subsection{In-gel digestion of P. monodon TGH and peptide mass finger- printing}

After manually excised from the polyacrylamide gel, the electrophoresis band of P. monodon TGH was cut into pieces, which were dehydrated with acetonitrile for $10 \mathrm{~min}$, vacuum dried, re-hydrated with $100 \mathrm{mM}$ dithioerythritol in $25 \mathrm{mM} \mathrm{NH}_{4} \mathrm{HCO}_{3}(\mathrm{pH} 8.5)$ at $37{ }^{\circ} \mathrm{C}$ for $1 \mathrm{~h}$. They were subsequently

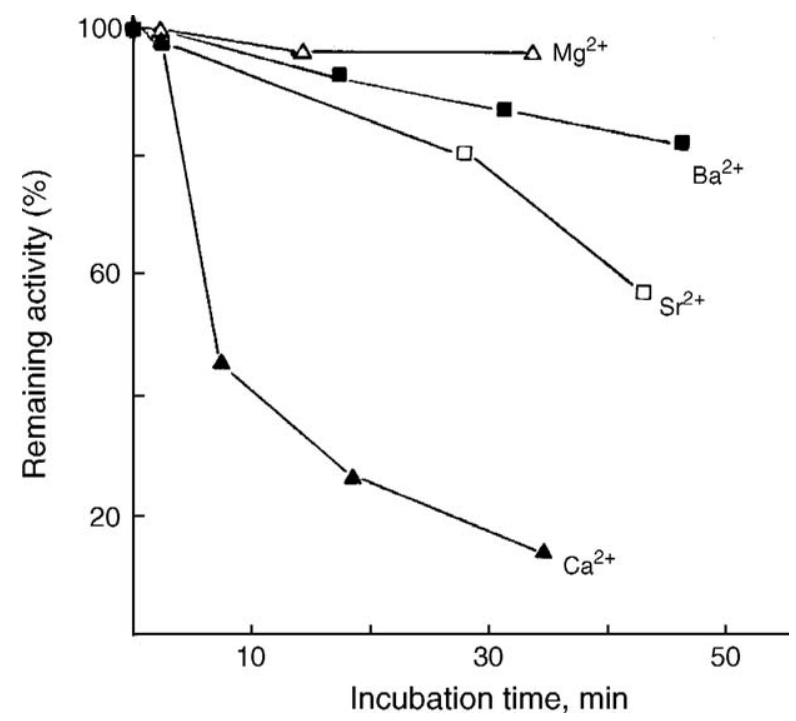

Fig. 5. Effects of metal chlorides on the enzymatic activity of P. monodon TGH. The hemocyte lysate $(0.4 \mathrm{mg}$ protein $/ \mathrm{ml}$ in $50 \mathrm{mM}$ Tris- $\mathrm{HCl}, 0.1 \mathrm{mM}$ ATP, $0.1 \mathrm{mM}$ EDTA, pH 7.5) was preincubated with $5.3 \mathrm{mM}$ metal chlorides at $4{ }^{\circ} \mathrm{C}$ $\left(\triangle-\triangle, \mathrm{MgCl}_{2} ; \boldsymbol{\square}-\mathbf{\square}, \mathrm{BaCl}_{2} ; \square-\square, \mathrm{SrCl}_{2} ; \mathbf{\Delta}-\mathbf{\Lambda}, \mathrm{CaCl}_{2}\right)$. Aliquots of sample were withdrawn at the time indicated and amine-incorporation activity of TGH was measured at $23{ }^{\circ} \mathrm{C}$. 
alkylated with $65 \mathrm{mM}$ iodoacetamide in $\mathrm{NH}_{4} \mathrm{HCO}_{3}$ at $27^{\circ} \mathrm{C}$ for $1 \mathrm{~h}$ in the dark, washed twice with $50 \%$ acetonitrile in $\mathrm{NH}_{4} \mathrm{HCO}$, dehydrated with acetonitrile for $10 \mathrm{~min}$ and dried, then hydrolyzed with $25 \mathrm{ng}$ modified trypsin (Promega, Madison, USA) in $25 \mathrm{mM} \mathrm{NH}_{4} \mathrm{HCO}_{3}(\mathrm{pH} 8.5)$ at $37{ }^{\circ} \mathrm{C}$ for $16 \mathrm{~h}$. The oligopeptides were extracted twice with $50 \%$ acetonitrile containing $5 \%$ formic acid for $15 \mathrm{~min}$ and dried under vacuum. Their masses were analyzed with MALDITOF/TOF and matched by Mascot Search programs.

\section{Results}

\subsection{Purification and characterization of P. monodon TGH}

Enhancement of the substrate fluorescence upon incorporating dansylcadaverine into succinyl casein was measured to assay

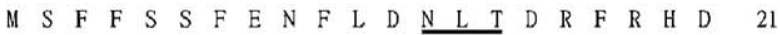

1 TCTTGAAGCGGATCGGTCCTTGGGGTTTTCTOCAAGATGTCGTTCTTCAGTTCCTTCCAGAATTTCCTGGACAACCTGACCGACOCCTTCAGGCATGAOC L E V N R R E D R E I E I A D E L 101 TCGAGGTCAACCGCCGCGAGGACAGGGAGATCGAGATTGCGGATGAACTTAATGATACAGCCAATGAAGTOGACTCOCGCGACGCCAACCCOGGAGGTGCT $\begin{array}{llllllllllllllllllllllllllllllllll}S & V & D & F & Q & A & K & P & N & A & V & N & H & F & C & D & K & Y & E & I & V & E & R & Q & Q & A & A & I & V & L & R & R & G & 88\end{array}$

201 GAGCGTGGACTTCCAGGCCAAACCGAATGCOGTGAACCACTTTTGCGACAAGTACGAAATCGTCGAGCGGCAGCAAGCGGCTATOGTCCTTCGCAGGGGG $\begin{array}{llllllllllllllllllllllllllllllllll}G & S & F & T & I & E & V & E & F & N & L & E & L & E & L & K & K & T & H & L & V & K & L & Y & F & S & F & G & Q & R & P & S & A & 121\end{array}$

301 GGGAGCTTCACCATCGAGGTGGAGTTCAACCTGGAGCTGGAGCTGAAGAAGACCCATTTGGTGAAGCTCTATTTTTCTTTTGGTCAGCGTCCGAGCGCGG $\begin{array}{llllllllllllllllllllllllllllllllllll}A & K & G & T & Q & A & A & L & Q & V & S & G & K & K & L & F & D & K & N & H & E & E & W & D & V & R & V & D & G & Q & N & G & K & K & 155\end{array}$

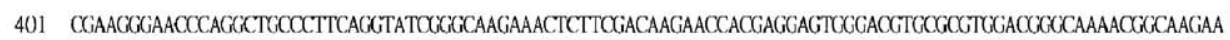

$\begin{array}{llllllllllllllllllllllllllllllllll}I & T & F & E & I & Q & I & P & T & D & A & P & V & G & V & W & N & P & A & V & E & V & S & L & R & A & N & S & D & T & T & R & H & 188\end{array}$ 501 AATCACCTTCGAAATTCAGATCCCCACCGACGCCOCCGGTACGTGTTTGGAATCCCGCGGTGGAAGTGAGTCTCAGAGCTAACTCGGACACGACCCGACAT $\begin{array}{llllllllllllllllllllllllllllllllll}\mathrm{L} & H & \mathrm{R} & \mathrm{S} & \mathrm{D} & \mathrm{T} & \mathrm{H} & \mathrm{V} & \mathrm{Y} & \mathrm{I} & \mathrm{L} & \mathrm{F} & \mathrm{N} & \mathrm{P} & \mathrm{W} & \mathrm{N} & \mathrm{K} & \mathrm{H} & \mathrm{D} & \mathrm{G} & \mathrm{T} & \mathrm{Y} & \mathrm{M} & \mathrm{P} & \mathrm{D} & \mathrm{E} & \mathrm{E} & \mathrm{K} & \mathrm{R} & \mathrm{D} & \mathrm{E} & \mathrm{Y} & \mathrm{V} & 221\end{array}$

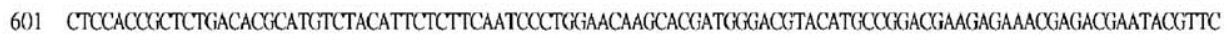

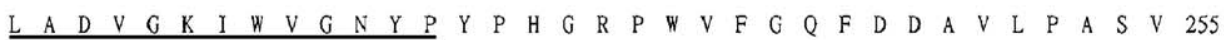

701 TGGCTGACGTAGGAAAGATCTOGGTGGGTAACTAOCOCTACCOCCACGGTOCOCOCTGGGTCTTCOCCCAGTTOGAOGAOCOCGTOCTGCCGGCGAGCGT $\begin{array}{llllllllllllllllllllllllllllllllll}\text { L } & L & L & E & K & S & G & V & P & P & E & S & \underline{R} & G & D & P & I & R & V & S & R & A & I & S & K & M & V & N & S & N & D & D & Q & 288\end{array}$ 801 GCTGCTGCTCGAGAAGTCGGGCGTCOCGCCGGAGTCTOGCGGCGACCOCATCOGCGTGTCCAGAGCCATCTOCAAGATGGTGAACAGCAACGACGATCAG $\begin{array}{llllllllllllllllllllllllllllllllll}G & V & I & V & G & R & W & D & G & K & Y & E & D & G & K & S & P & S & S & W & S & G & S & I & K & I & L & E & E & Y & I & K & S & 32 I\end{array}$

901 GGCGTGATCGTGGGTCGTTGGGACCGCAAGTACGAAGACGGGAAGTCOCOCTOGTOGTGGTCAGGCTCCATCAAGATCCTGGAGGAATACATCAAAAGCA

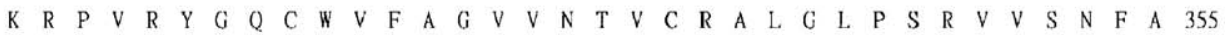
1001 AGCGCCCGGTCCGCTACGGCCAGTGCTGGGTCTTOCCOGGGGTTGTCAACACAGTGTGTOGTGCTCTCGGTCTGCCCAGTCGAGTGGTCAGCAACTTCGC

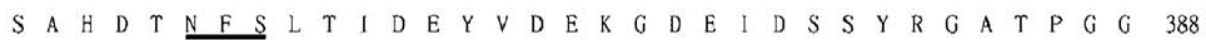
1101 CTCAGCACACGACACCAACTTCTCCCTCACCATTGACGAATACGTTGACGAGAAGGGOGAOGAGATCGACTCCTCATACOGCGGTGCTACCOCTGGAGGC $\begin{array}{llllllllllllllllllllllllllllllllll}L & Y & D & S & I & W & N & F & H & V & W & N & D & V & W & M & I & R & P & D & L & P & Q & G & Y & S & G & W & Q & V & I & D & A & 421\end{array}$

1201 CTGTATGATTCCATCTGGAACTTCCACGTTTGGAATGACGTGTGGATGATCCGACCTGACCTGCCACAGGGTTACAGCGGCTGGCACGTCATCGACGCAA

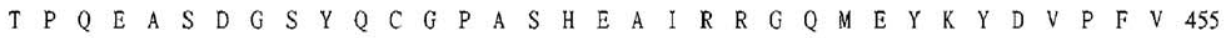
1301 CGCCACAGGAAGCAAGTGATGGCTOCTACCAGTGTGGGCCAGCGTCCCAOGAGGCCATCCGCAGAGCCCAAATGGAATACAAGTACGACGTGCCCTTCGT

$\begin{array}{llllllllllllllllllllllllllllllllll}L & A & E & V & N & A & D & V & V & H & W & Q & K & D & D & K & A & Q & D & G & F & K & K & L & T & T & N & K & R & S & V & G & R & 488\end{array}$

1401 GCTGGCCGAGGTCAACGCTGACGTCGTGCACTGGCAGAAGGACGATAAGGCACAGGACGGGTTCAAGAAGCTCACCACGAACAAGAGGAGCGTCGGCCGC $\begin{array}{llllllllllllllllllllllllllllllllll}\text { Q } & V & L & T & K & Q & A & G & P & S & G & D & K & E & D & I & T & L & E & Y & K & P & K & E & G & T & T & A & E & R & V & T & L & 521\end{array}$

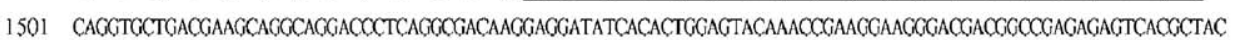
$\begin{array}{lllllllllllllllllllllllllllllllllll}\mathrm{L} & \mathrm{T} & A & A & \mathrm{R} & \mathrm{R} & \mathrm{T} & \mathrm{R} & \mathrm{A} & \mathrm{A} & \mathrm{R} & \mathrm{H} & \mathrm{A} & \mathrm{F} & \mathrm{R} & \mathrm{L} & \mathrm{M} & \mathrm{S} & \mathrm{Q} & \mathrm{G} & \mathrm{V} & \mathrm{D} & \mathrm{D} & \mathrm{V} & \mathrm{E} & \mathrm{F} & \mathrm{G} & \mathrm{L} & \mathrm{E} & \mathrm{D} & \mathrm{I} & \mathrm{E} & \mathrm{K} & \mathrm{V} & 555\end{array}$ 1601 TAACAGCTGCCOGAAGGACOCGOGCOGCCAGACAOGCCTTCOGTCTCATGTCOCAAGGAGTAGATGACGTTGAGTTOGGOCTOGAGGACATCGAGAAAGT

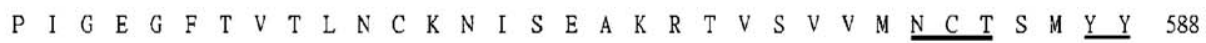

1701 GCCCATCGGCGAAGGGTTCACCGTGACGCTCAATTGCAAGAACATTAGCGAGGCCAAGAGAACGGTGAGTGTGGTAATGAACTGCACCAGTATGTACTAC $\begin{array}{llllllllllllllllllllllllllllllllll}T & G & A & P & A & Y & P & I & K & R & R & E & G & E & I & V & L & E & V & G & E & T & K & N & M & T & M & E & V & T & Y & G & D & 621\end{array}$

1801 ACAGGCGCCCCCGCTTACCOGATAAAGCGCOCTGAGGGAGAAATCGTTCTOGAAGTTGGAGAAACCAAGAACATGACCATGGAAGTGACGTACGGTGACT Y $Y$ Y E $K$ K L $L$ V E 1901 ACTACGAGAAACTGGTCGAACACGCCATGGTAAAGAACGTGGCTATTTGCACCGTGGCGGAAACCTCTTACGCGTGGGTGGGCGACGATGGCATGGAGAT

$\begin{array}{llllllllllllllllllllllllllllllllll}\text { Q } & K & P & D & I & K & I & E & I & L & S & E & A & T & A & G & L & P & L & A & L & R & I & S & F & T & N & P & L & P & I & T & L & 688\end{array}$ 2001 ACAGAAGCCGGATATCAAGATCGAGATCCTGTCGGAGGOCACTGCGGGATTACCOCTOGCCCTCAGGATATCCTTCACCAATCCOCTGCOGATAACOCTC $\begin{array}{llllllllllllllllllllllllllllllllll}R & N & C & E & L & E & V & D & G & P & G & I & L & R & P & K & T & I & P & I & S & N & V & G & P & S & E & N & M & V & H & E & M & 721\end{array}$ 2101 AGGAACTGCGAGCTGGAGGTGGATGGTCCCGGCATCCTGCGCCCCAAGACTATACCGATCAGCAACGTGGGCCCCAGTGAGAATATGGTCCATGAGATGA K V V F 2201 AAGTGTTCCCAAAGAAACOCAAGCACTGCACAATAGTOGTGACCTTCAACTCCAGCCAGCTGTACAACCTTACGGGGTCAACTCAGGTCACCATCAATOC A E * 757

2301 GGCAGAGTGATCGCGTGGTGATTTTGTGAGCTGCAAGGaAGGATTTTGAaGAaTGCTATCTATCACTCCTCTGGAaAAAAAAAAAAA

Fig. 6. The cDNA and deduced amino acid sequence of PmTG. Sequences homologous to those of Mj-TGH peptides purified by HPLC are underlined. Potential Nglycosylation sites and the RGD motif are doubly underlined. 
TGH during purification (Fig. 1). The yield was high (0.6 mg $\mathrm{TGH}$ from $7.6 \mathrm{mg}$ of the total hemocyte lysate proteins). Increase of the specific activities of TGH after the two purification steps was about 4-folds. The activity was hardly detected in the shrimp plasma. The molecular mass of the purified TGH was determined as $84280 \pm 168$ Da by MALDITOF mass spectrometry (Fig. 2A). However, the molecular weight measured by a gel filtration column was about $170-$ $180 \mathrm{kDa}$ (Fig. 2B), and it was estimated to be about $85-90 \mathrm{kDa}$ by SDS-PAGE under both reducing and non-reducing condition (Fig. 2C). Thus, the TGH most likely existed as non-covalent homodimers, which could be dissociated in the presence of SDS during electrophoresis. We found that TGHs of both shrimps were N-terminal blocked, thus no results could be obtained by automatic sequencing. Presumably like many other transglutaminases, the $\mathrm{N}$-terminus was acetylated after removal of the initiator-methionine residue. This acetylation possibly serves as a signal for protein secretion [1].

\subsection{Enzymatic properties of P. monodon TGH and the effect of $\mathrm{Ca}^{2+}$}

We noticed that the amine-incorporating activity of purified TGH and its hemolymph-clotting activity were well correlated (Fig. 3A). Like other member of group II transglutaminase family, $\mathrm{Ca}^{2+}$ is an essential cofactor or specific activator of shrimp TGH. The half-maximum concentration of $\mathrm{Ca}^{2+}$ required for the incorporation of dansylcadaverine of P. monodon TGH $\begin{array}{lllllllllllllllllllllllllllllllllll}N & D & L & E & V & C & R & R & E & D & R & D & L & E & I & E & N & E & L & N & E & I & T & R & A & F & S & A & S & K & E & P & K & I\end{array}$

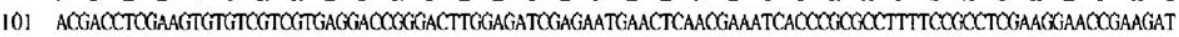

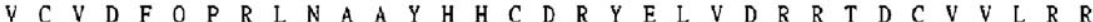

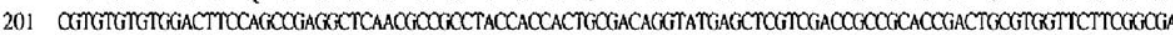
G $G$ G

301 GGTGGTATGTTCTCCATGGTTGTCGAACTCAACCAGCAAGTGAACCTTTCGCCOGAACAGCAGCTCAAGCTCTACTTCTCCTTCGGACCTCGCCOCAACG

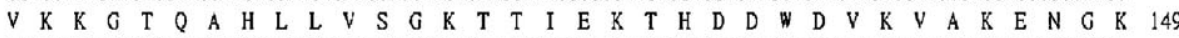
401 TAAAGAAGGGCACGCAAGCGCACCTCCTCGTCTCAGGCAAGACAACGATTGAAAAAACACATGAOGATTGGGACGTGAAGGTCGOCAAGGAAAACGGGAA

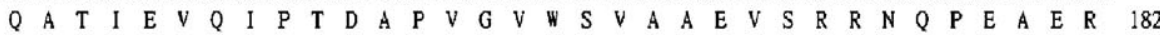

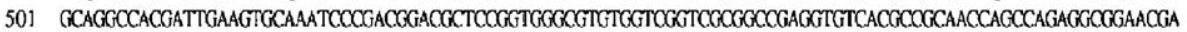

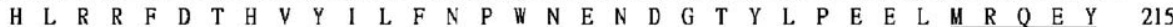

601 CAOCTGCGCOGCTTCGACACOCATGTCTACATCCTCTTCAAOCCTTGGAATGAGAATGACGGAACATAOCTTCCOGAGGAATTAATGCGACAGGAGTATG $\begin{array}{lllllllllllllllllllllllllllllllllll}V & L & E & D & V & G & K & V & F & V & G & S & Y & P & H & T & R & G & R & H & W & A & F & G & Q & F & D & D & A & V & L & P & A & C & 249\end{array}$ 701 TGCTTGAGGAOGTAGGCAAGGTGTTOGTOGCCAGTTACOCTCACACTOCACGTOCCCACTGCGOCTTCGGGCAGTTOGAOGACGCTGTGCTTOCGCOCGTG $\begin{array}{lllllllllllllllllllllllllllllllllll}M & L & L & L & E & R & S & G & V & K & A & E & A & \underline{R} & G & D & P & V & R & V & S & R & A & L & S & K & M & V & N & S & N & D & D & 282\end{array}$ 801 CATGTTGCTGCTOGAGAOGTCAGCCGTCAACGCOGAGGCTOGAOCCGAOCOOGTGCCCGTGTOGAGGGCOCTGTOCAAGATOGTGAACAGTAACGACGAC

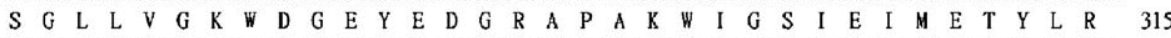

901 TCOGGCCTTCTCGTCGGAAAATCGGACGGCGAGTACGAAGAOCGCAGACCACCAGCGAAGTGGATOGGCTCCATCGAAATCATGGAGACATACCTTAGGA $\begin{array}{lllllllllllllllllllllllllllllllllllll}T & R & 0 & S & V & K & Y & G & O & C & W & V & F & A & A & C & L & N & T & I & C & R & A & L & G & M & P & C & R & V & V & T & N & F & 349\end{array}$ 1001 OCAGGCAGTCIGTTAAGTACGGACAGTGTTGGGTCTTCGCOGCCTGCCTCAACACGATCTGTCGOCCTCTGGGAATGCCATGCOGAGTCCTGACCAATTT $\begin{array}{lllllllllllllllllllllllllllllllllll}\text { A } & S & A & H & D & T & N & \text { I } & S & L & P & I & D & E & Y & F & D & E & D & G & D & K & I & E & E & N & D & R & Y & A & D & P & A & 382\end{array}$ 1101 CGOCTCTGCACACGACACCAACATTTCOCTOCOCCATCGACGAGTACTTIGATGAGGACGGTGATAAGATCOGAGGAGAACGATCGCTACGOCGGCCCOCGCT $\begin{array}{llllllllllllllllllllllllllllllllll}G & I & R & D & S & I & W & N & F & H & V & W & N & D & V & W & M & S & R & P & D & L & P & D & G & F & G & G & \mathbb{N} & Q & V & I & D\end{array}$

1201 GGTATTCOTGACTCCATCTGGAACTTCCACCTCTGGAATGACGTGTGGATGAGCCGACCTGACCTTCCOGACGGTTTCGGAGCCTGGCAGGTCATCGACO

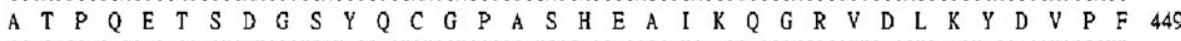
1301 OCACACCACAGGAGACGAGTGATGGGTCGTACCAGTGTGGTOCOCCATCOCACGAGGCCATCAAGCAGGGTOGCGTTGACCTGAAGTACGACGTGCOCTT

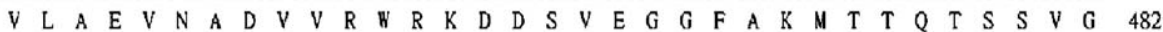
1401 OGTGCTGGCCGAGGTCAACGOCGACGTOGTGCOCTGGCGGAAGGACGACTCGGTTGAGGGAGGATTOGOGAAGATGACAACGCAGACGAGCAGCGTGGGC

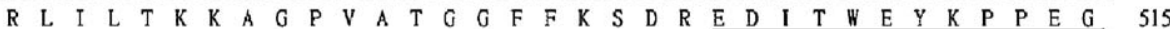

1501 CGTCTGATCCTGACGAAGAAGGCCGGACCAGTGGCCACAGCGCGCTTCTTCAAGTCCGATCGGGAAGACATCACGTOGGAGTACAAGCCTCCOGAGGGAA $\begin{array}{llllllllllllllllllllllllllllllllllll}T & R & A & E & R & V & S & I & L & T & A & A & R & R & T & R & T & A & R & Q & V & F & D & M & P & S & E & A & K & E & D & I & V & F & 549\end{array}$

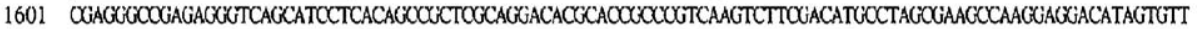

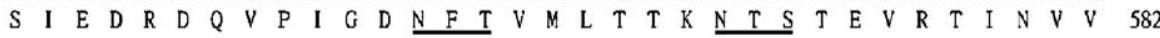
1701 CAGCATTGAAGATCGIGACCAGGTTOCTATOGGOGACAATITCACCGTGATGTTAACCACCAAGAACACGACCAOCCGAAGTGAGGACGATCAACGTGGTG $\begin{array}{lllllllllllllllllllllllllllllllllll}M & T & C & A & S & M & Y & Y & T & G & A & K & A & H & T & \text { I } & \text { K } & \text { R } & H & \text { D } & \text { G } & \text { E } & \text { I } & \text { H } & \text { L } & \text { E } & \text { P } & \text { N } & \text { O } & \text { T } & \text { K } & \text { T } & \text { M } & 615\end{array}$

1801 ATGACGTGCGOCAGCATGTACTACAOCGGACCCAAAGCOCACACCATCAAACGOCATGACGGGGAGATACACCTGGAGCOCAATCAGACCAAGACTATGT $\begin{array}{llllllllllllllllllllllllllllllllllll}S & M & E & V & Y & Y & S & D & Y & Y & S & K & L & V & D & H & D & \text { L } & \text { I } & K & \text { S } & \text { I } & \text { V } & \text { I } & C & N & \text { V } & \text { N } & \text { E } & \text { T } & \text { T } & 0 & C & W & 649\end{array}$

1901 OCATGGAGGTGTACTACTCTGACTACTACTOCAAGCTGGTGGAOCATGACCTTATCAAGAGCATTGTGATCTGTAAOGTCAATGAAACTACACAATGCTG

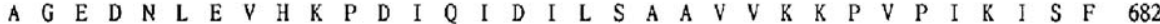
2001 GGCOGGOGAAGACAACCTIGAAGTCCACAAACCTGACATCCAGATTGACATCTTGTCACOCGCCOGTGGTGAAGAAGCOCGGTTCOCATCAAGATATCCTTC

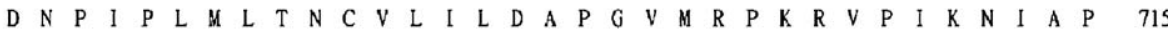

2101 GACAACCCTATTCCCCTGATGTIGACGAATTGCGTTTIGATACIGGACGCTCCIGGAGTTATGCGCOCTAAGAGAGTGCCTATCAAAAACATCGCCCCAA

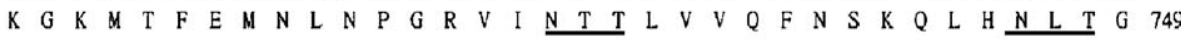
2201 AGGGAAAGATGACTTTTGAGATGAACCTGAACCOGGGGAGGGTAATAAATACTACTCTOGTOGTGCAATTCAACAGCAACCAACTGCACAATCTCACTGG A $K$ K $K$ K V V V V $S$ S P A *

2301 TGCCAAGAAGGTTGTTGTTTCCCCCOCTTGAAGAGCGTGGTAAGGGAACGCACCCCCACATGGCTTGAGAAATGAGAGGAGTCAGAAGTCGCOGAGTGTT 2401 TGATCGAAATGTATCACTGTTTTCTTCTTCTCCTCATTCTCOCTCTOGTTCTTCTTGTOCTOCTTTTCCTTCTCCTTATTCTATTTTTTCTGTTTTTCCT

2501 TGACTTAAACGACCTGCTGAGCCAAAGAGAGAAACCTTTTCCTGTTTGTTGTTTTACGCTTATTTGTGGCAATGATTAGCTACTGGTAATGTTGGAGCAG 2601 TAGCTACCACGTAGGGACTAAGAGTTGCATCCTGTTTACGAATTTTCCAAGAGCTTTGGGAATAAGTATGCGAAATOCTTTTTGATATTCGAATATGTTT 2701 GCTTCAGTTTGAATTGTATGAGTAAATGGAGTAAACTACAATAGCTCATTTTGTTGTTAGCGTAATATACTACATAGCCCAGTCACATGGCCAAATTGGA 2801 AGAATGTTTAACAGAAATATTTATATTGCATATGTCACTTGTAACOCTGAAAAAATATGGTAGGTTTAAGACAAAACCGTAGTTATGTAATTACTGTTGT 2901 CTTGACTCTTGCTTGCACAAAACGATAAAGGTCATATGGAATTAGTTTGTGAAAAGCAATAAAACGAAAAAAAAAAAAAAAAAAAAAAAAAAAAAAA

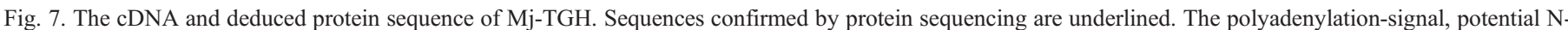
glycosylation sites and the RGD motif are doubly underlined. 
was about $3 \mathrm{mM}$ (Fig. 3B). Similar level of $\mathrm{Ca}^{2+}$ was required for clotting of hemolymph by TGH. This is consistent with the normal $\mathrm{Ca}^{2+}$ requirement of other transglutaminases [1]. Other metal chlorides, e.g., $10-100 \mathrm{mM}$ of $\mathrm{SrCl}_{2}, \mathrm{BaCl}_{2}$ or $\mathrm{MgCl}_{2}$, could not substitute $\mathrm{CaCl}_{2}$ in both TGH assays (data not shown). The strict dependency on $\mathrm{Ca}^{2+}$ is different from that for Factor XIII, which can be activated by other divalent metal ions although less effectively [26].

MJ-TGH IPTVNPYN----ELINRFTNDLEVCRREDRDLEIENELNEITRAFS-ASKEPKIVCVDFQPRLNAAYHHCDRYELVDRRTDCVVLR 81

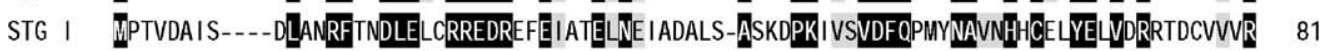

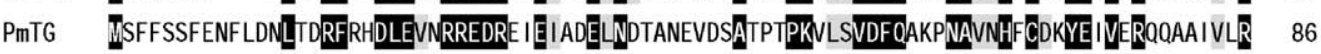

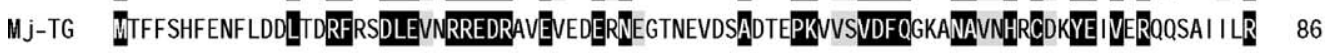

Mj-TGH RGGMFSMVVELWQQVNLSAEQQLKLYFSFGPRPNVKKGTQAHLLVSGKTTIEKTHDDWDVKVAKENGKGATIEVQIPTDAPVGWWS 167 STG I RGGLLSMVVKLIONVALASAQELKFVFSFGSRPNVEKGTQAHLVVTGKTKIEKPHDDWDIRLVKEAGKKATVEVQIPTDAPVGVWKK 167

PmTG RGGSFTIEVEFWLELELKKTHLVKLYFSFGORPSAAKGTQALLOVSGKKLFDKNHEEWDVRVDGONGKKIIJFEIQQIPTDAPVGVWN 172

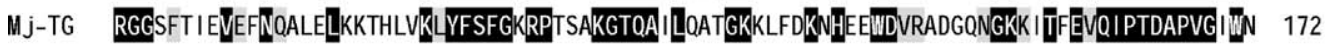

Mj-TGH VAAEVSRRNOPEAERHLRRFDTHYYILFNPWNENDGTYLPEELMROEYVLEDVGKVFVGSYPHTRGRHWAFGQFDDAVLPACMLLL 253

STG I VAAEVTLRNQ-AGERHLRRFDTMVYILFNPUNENDGTYLPEEKMROEYVLSAVGTVFVGSYPHTRGRHWAFGQFDDAVLPACMLLL 252

PmTG PAVEVSLRANSDTTRHLHRSDTHVYILFNPUNKHDGTYMPDEEKRDEYVLADVGK IWVGNYPYPHGRPWFGQFDDAVLPASVLLL 258

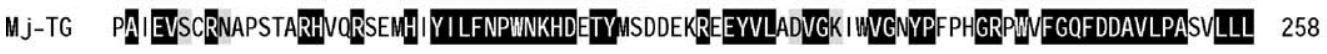

Mj-TGH ERSGVKAEARGDPVRSRALSKMVNSNDSGLLVGKWDGEYEDGRAPAKWIGSIEQWETYLRTRQSVKYGOCWVFACLVTICRAL 339 STG I ERSGVSAEARGDPVRSRALSKMVNSNDDSGLLVGKWDGEYEDGRAPAKVILSIEDLEMYLRTRQSVKYGOCWVFAACLNTICRAL 338 PmTG EKSGVPPESRGDPIRVSRAISKMVNSNDDDGVIVGRWDGKVEDGKSPSSWWGSIKDLEEVI KSKRPVRVGOCWVFAGVVNTVCRAL 344 Mj-TG ERSGIPSESRGDPIRVSRAISKMVNSNDDSGV IVGRWDGKVEDGKSPSSWSGSIKDLEAVI KSKRPVRVGQCWVAGVVNTVCRAL 344

Mj-TGH GWPCRVWTNFASAHDTN।SLPIDEYFDEDGDKDEENDRYADPAGIRDSIWNFHWWNDWMSRPDLPDGFGGWQVIDATPQETSDGS 425 STG I GLPCRWTNFASAHDTNISLLSIDEYFDEDGDDIDAADRYVNPAGI RDS IWNFFNWNDVWISRPDLPDGFGGWQVIDATPQETSDGS 424 PmTG GLPSRWVSNFASAHDTNFSLTIDEYDEKGDEDDSSYRGATPGGLYDSIWNFFNWNDVWM IRPDLPOGYSGWOVIDATPQEASDGS 430 Mj-TG GLPSRWSSFFASAHDTMSLLIDEWDEKGNEIDSSYTGATPGGLYDS IWNFFNWNDVWMMPDLPEGFGGWQVIDATPQETSDGT 430

Mj-TGH YQCGPASHEAIKOGRVDLKYDVPFVLAEVNADVVRWRDDSVEGGFAKMTTIOTSSVGRLILTKKAGPVATGGFFKSDREDITWEYK 511 STG I VQCGPASHEAIROGRVDLKYDVPFVLAEVNADVVRVIIKDDRAEGGFKKLATITSSVGRLILTKKAGPVATGGFFKSDREDITWEYK 510 PmTG VQCGPASHEAIRRGOMEYKYDVPFVLAEVNADVVHWOKDDKAQDGFKKLTTNKRSVGRQVLTKQAGPS-G-..--DKEDITLEYK 509 Mj-TG VQCGPASHEAIRRGQMEYKYDVPFVLAEVNADVVHWOKDDKAKDGFKKLTTINKRSVGRQLLTKKPGPVDNSGYSGTDKEDITLEYK 516

Mj-TGH PPEGTRAERVSILIAARRTRTARQVEDMPSEAKEDIVESIEDRDQVPIGDNFTVMLTTKNTSTEVRTIMVVMTCASMYYTGAKAHT 597 STG I PPEGTRAERVSILNAARRTRTARQVEDMPSEAKEDVTFRIEDRDOVPIGDNFSVT I TATNTSSEVVTIIMVVMTCASMYYTGAKAHT 596 PmTG PKEGTTAERVTLLIAARRTRAARHAFRLISOGVDDVEFGLEDI EKVPIGEGFTVTLNCKNISEAKRTVSVVMNCTSMYYTGAPAYP 593 Mj-TG PKEGTTAERVTLLIAARRTRAARHAFRLISOEVDDVEFTLEDLERVPIGEGFTVTLTAKMUSEAKRTISVVMTCSSMYYTGAPAFP 600

MJ-TGH IKRHDGEIHLEPNOTKTWSWEVYYSDYYSKLVDHDLI KS IVICNVNETTOCWAGEDNLEVHKPDIODDILSAAVVKKPVPIKISFD 683 STG I IKRHDGELHLEPSOTKTWSWEVYYSDYYSKLVDHDLI KS IVICNVNETTOCWGGEDNLEVHKPDINDEVLGSAVEGKAVPI KVSFD 682 PmTG IKRREGEIVLEVGETKNMTMEVTYGDYYEKLVEHANKNVAICTVAETSYAWGGDGMEIOKPDIKDEILSEATAGLPLALRISFT 679 Mj-TG IKRREGEIVLGVGETKNQTMEVTHGDYYEKLVEHAFVRNVAICTVSETSYAWGDDGLEI IKPDVKDEILSEAVAGKPLPIRVSFT 686

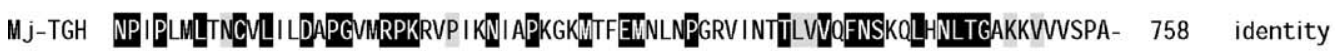

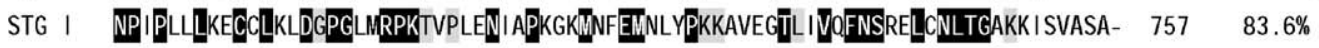

PmTG NPLPITLRNGELEVDGPGILRPKTIPISWVGPSENMWHEMKVFPKKPKHCTIVWTENSSOLIVLTGSTQVTI INPAE $757 \quad 58.8 \%$

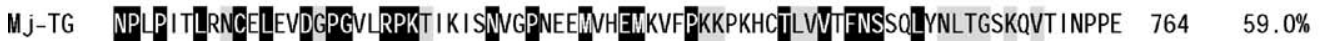

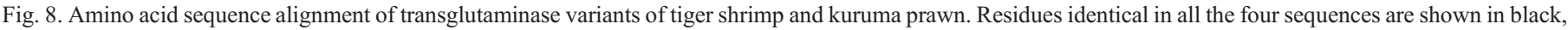

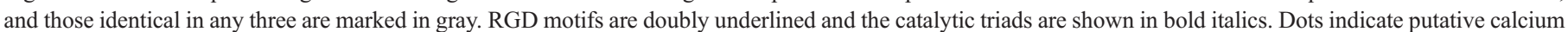
binding sites, and sequences matching those of the purified Mj-TGH oligopeptides are underlined. 


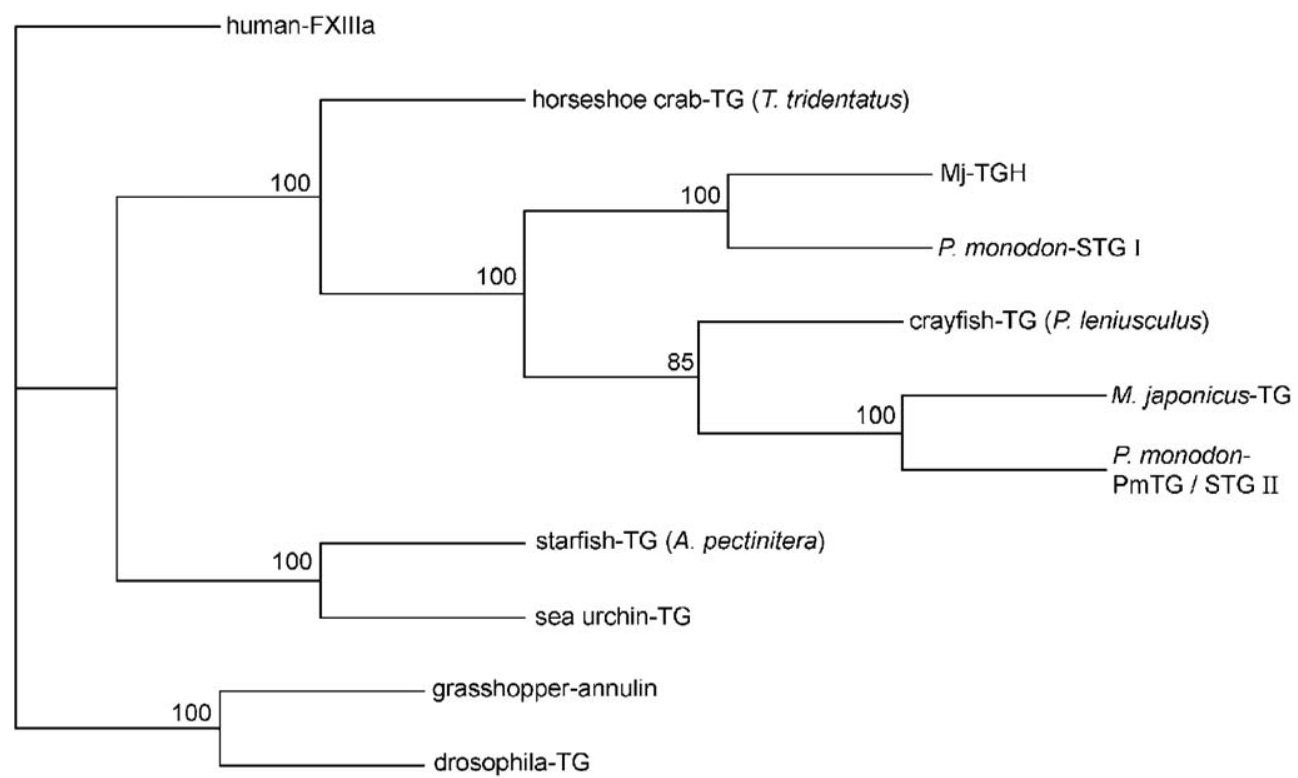

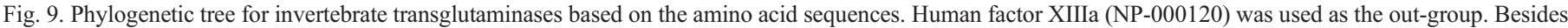

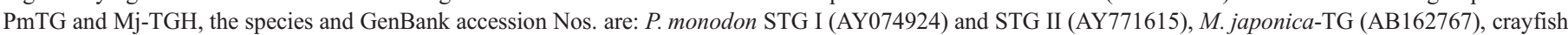

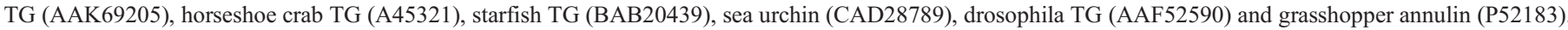
Bootstrap values are shown at the nodes.

P. monodon TGH appeared to be labile at temperature $>25^{\circ} \mathrm{C}$ and $\mathrm{pH}$ outside the range 7-9 (Figs. $4 \mathrm{~A}$ and $\mathrm{B}$ ). Lyophilization or frozen-thaw could inactivate the enzyme by more than $50 \%$. However, more than $85 \%$ of the activity could retain for several days if purified TGH or the hemocyte lysate was stored in the presence of metal chelator (e.g., EDTA) at $\mathrm{pH} 7-9$ at $0-4{ }^{\circ} \mathrm{C}$, or kept in buffer in the presence of EDTA and 35\% (v/v) glycerol below $-14{ }^{\circ} \mathrm{C}$.

Like other type II transglutaminases, $P$. monodon TGH was inhibited or inactivated by certain divalent metal ion (e.g., 10$20 \mu \mathrm{M} \mathrm{ZnCl} 2$ ) which competed $\mathrm{Ca}^{2+}$ binding [3,26,27], thiolreactive agents (e.g., organomercurials and iodoacetamide), or 2-10 $\mathrm{mM}$ histamine and other primary amines (data not shown). Like other transglutaminases $[1,28]$, addition of $5 \mathrm{mM} \mathrm{Ca}^{2+}$ to the medium greatly facilitated the inhibition of TGH by $3-$ $10 \mu \mathrm{M}$ mercurial or $0.1 \mathrm{mM}$ iodoacetamide (data not shown), suggesting exposure of the TGH active site by a $\mathrm{Ca}^{2+}$ induced conformational change. Notably, TGH activity was decayed in minutes at $\mathrm{mM}$ concentrations of $\mathrm{Ca}^{2+}$. Addition of EDTA could stop the $\mathrm{Ca}^{2+}$-induced decay immediately but could not reverse it, and the presence of dithiothreitol in the enzyme buffer only weakly protected this decay (data not shown). Moreover, preincubation with other metal chlorides also inactivated the enzyme although less effectively than $\mathrm{CaCl}_{2}$ (Fig. 5).

\subsection{Cloning, sequencing and phylogenic analysis}

Automatic sequencing of two oligopeptides obtained by HPLC-purification of Lys-C digest of Mj-TGH showed their amino acid sequences as MRQEYVLSDVGTVFVGSYP and EXLTWEYKPPEGTRAXRVSILN, respectively. The sequence of another purified CNBr cleavage product was YYTGVPAH TIKRHDGELHLEPSQT. Degenerate PCR primers were de- signed based on these $\mathrm{Mj}$-TGH partial sequences. In addition to the cDNA encoding STG I (AY074924), a 578-bp cDNA encoding the C-terminal part of a novel P. monodon transglutaminase (designated as PmTG) was produced by PCR. On the other hand, a $1071 \mathrm{bp}$ cDNA encoding the $\mathrm{C}$-terminal part of $\mathrm{Mj}$-TGH was also amplified. The full-length cDNA of PmTG and Mj-TGH were then completed by $5^{\prime}$ RACE. PmTG had a total length of $2,387 \mathrm{bp}$, including $36 \mathrm{bp}$ of the $5^{\prime}$-untranslated region, an open reading frame of $2,271 \mathrm{bp}$, and $81 \mathrm{bp}$ of the $3^{\prime}$-untranslated
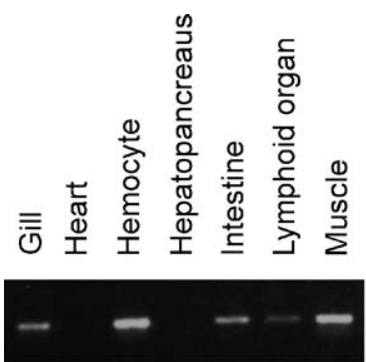

25 cycles

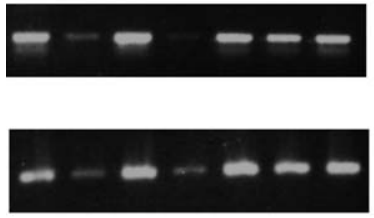

30 cycles

35 cycles

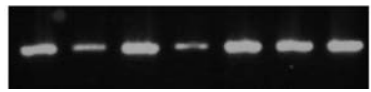

40 cycles

Fig. 10. RT-PCR analysis of tissue expression of PmTG. Total RNA was extracted from various tissues of P. monodon. Specific primers were designed to amplify a 390-bp fragment of PmTG cDNA. PCR products at various cycles were analyzed by electrophoresis on $1.2 \%$ agarose gel containing ethidium bromide. 
region. The putative initiating ATG codon, according to Kozak's rule [29], is at nucleotide 33 (Fig. 6). The open reading frame of PmTG is predicted to encode a protein of 757 residues (pI 5.47) and similar to STG II [10]. Its ORF differs from that of STG II by 15 nucleotides and resulted in the substitution of six amino acid residues, i.e., $\mathrm{L} 158 \rightarrow \mathrm{F}, \mathrm{T} 182 \rightarrow \mathrm{N}, \quad \mathrm{Q} 212 \rightarrow \mathrm{P}, \mathrm{V} 495 \rightarrow \mathrm{A}$, $\mathrm{S} 499 \rightarrow \mathrm{G}$, and $\mathrm{E} 541 \rightarrow \mathrm{G}$. None of them are in the catalytic core domain of the enzymes.

We also cloned Mj-TGH cDNA which contained 2996 bp (included $54 \mathrm{bp}$ of the $5^{\prime}$-untranslated region, an open reading frame of $2274 \mathrm{bp}$, and $669 \mathrm{bp}$ of the $3^{\prime}$-untranslated region). Its authenticity was confirmed by sequence analyses of the fragments obtained from $\mathrm{CNBr}$ cleavage and protease digestion of the purified M.japonicus TGH. The putative initiating ATG [29] is at nucleotide 55, and its open reading frame encodes an enzyme of 758 residues (pI 5.76) with seven potential $N$ glycosylation sites and one RGD motif (Fig. 7). The predicted Mj-TGH sequence is $83 \%$ and $59 \%$ identical to those of STG I and STG II/PmTG, respectively (Fig. 8). PmTG and Mj-TGH probably are both cytosolic, since they do not contain a typical signal-peptide or trans-membrane domains.

Amino acid sequences of all the invertebrate transglutaminases so far solved were aligned and a phylogenetic tree has been constructed to study their structural relationships (Fig. 9). All the Crustacean enzymes are linked together in the tree and closer to those of other marine arthropods than to those of insects. Apparently, STG I and Mj-TGH are orthologous to each other.

\subsection{RT-PCR analyses and tissue expression profile}

The expression patterns of PmTG mRNA in various tissues of P. monodon were analyzed by RT-PCR. As shown in Fig. 10, a 390-bp DNA fragment could be amplified in contrast to the negative control. The expression levels were highest in hemocyte and muscle, moderate in gill, intestine and lymphoid organ, and lowest in heart and hepatopancreas.

\subsection{In-gel digestion and mass analysis of TGH}

Purified P. monodon TGH band in gel was digested with modified trypsin before analyzed by peptide-mass-fingerprinting. The masses matched nicely with those of the peptides predicted from P. monodon STG I [8]. We found at least $34 \mathrm{TGH}$ peptides matching those of STG I (Table 1), representing a total of 396 amino acids or 52\% (396/757) of the entire sequence and evenly distributed.

Table 1

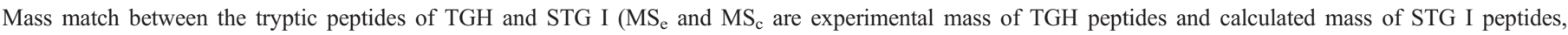
respectively)

\begin{tabular}{|c|c|c|c|}
\hline Tryptic peptides of STG I & position & $\mathrm{MS}_{\mathrm{e}}$ & $\mathrm{MS}_{\mathrm{c}}$ \\
\hline FTNDLELCR & $14-22$ & 1167.57 & 1167.52 \\
\hline EDREFEIATELNEIADALSASK & $24-45$ & 2450.13 & 2450.18 \\
\hline EFEIATELNEIADALSASK & $27-45$ & 2050.95 & 2050.01 \\
\hline EFEIATELNEIADALSASKDPK & $27-48$ & 2390.14 & 2390.19 \\
\hline IVSVDFQPMYNAVNHHCELYELV & $49-73$ & 3047.36 & 3048.41 \\
\hline RGGLLSMVVK & $82-91$ & 1058.62 & 1058.63 \\
\hline GGLLSMVVK & $83-91$ & 902.47 & 902.53 \\
\hline LNQNVALASAQELK & $92-105$ & 1497.73 & 1497.82 \\
\hline FYFSFGSRPNVEK & $106-118$ & 1576.77 & 1576.77 \\
\hline GTQAHLVVTGK & $119-129$ & 1109.60 & 1109.62 \\
\hline IEKPHDDWDIR & $132-142$ & 1422.68 & 1422.69 \\
\hline VAAEVTLR & $168-175$ & 857.49 & 857.50 \\
\hline FDTHVYILFNPWNENDGTYLPEEK & $186-209$ & 2940.31 & 2940.36 \\
\hline MVNSNDDSGLLVGK & $275-288$ & 1447.63 & 1447.70 \\
\hline WDGEYEDGR & $289-297$ & 1125.42 & 1125.44 \\
\hline WDGEYEDGRAPAK & $289-301$ & 1492.64 & 1492.66 \\
\hline WLGSIEILEMYLR & $302-314$ & 1621.82 & 1621.85 \\
\hline VVTNFASAHDTNISLSIDEYFDEDGDQIDAADR & $344-376$ & 3642.54 & 3642.62 \\
\hline YVNPAGIR & $377-384$ & 888.41 & 888.48 \\
\hline YDVPFVLAEVNADVVR & $444-459$ & 1804.91 & 1804.94 \\
\hline LATQTSSVGR & $473-482$ & 1018.53 & 1018.54 \\
\hline AGPVATGGFFK & $489-499$ & 1050.48 & 1050.55 \\
\hline SDREDITWEYKPPEGTR & $500-516$ & 2077.94 & 2077.97 \\
\hline VSILNAAR & $520-527$ & 841.45 & 842.50 \\
\hline EDVTFQIEDR & $544-553$ & 1250.56 & 1250.58 \\
\hline TINVVMTCASMYYTGAK & $577-593$ & 1908.83 & 1909.86 \\
\hline HDGELHLEPSQTK & $600-612$ & 1489.70 & 1489.72 \\
\hline LVDHDLIK & $627-634$ & 950.47 & 951.54 \\
\hline VSFDNPIPLLLK & $679-690$ & 1354.76 & 1354.79 \\
\hline LDGPGLMRPK & $696-705$ & 1081.62 & 1082.59 \\
\hline MNFEMNLYPK & $718-727$ & 1285.55 & 1285.58 \\
\hline KAVEGTLIVQFNSR & $728-741$ & 1560.84 & 1560.86 \\
\hline AVEGTLIVQFNSR & $729-741$ & 1432.74 & 1432.77 \\
\hline ELCNLTGAK & $742-750$ & 1005.52 & 1005.48 \\
\hline
\end{tabular}




\section{Discussion}

In shrimps, coagulation is initiated by activation of hyaline cells which release its content including the clotting enzymes [30-32]. P. monodon TGH effectively polymerizes shrimp clottable proteins to form stabilized gel (Fig. 3). Notably, the purified $P$. monodon TGH exists as weakly associated dimmers, like the A subunit of mammalian FXIII which is release from megakaryocytes as homodimers and also present as dimmers when crystallized. However, the shrimp TGH is possibly active as monomers like FXIII [1]. Dependent on seawater salinity, the $\mathrm{Ca}^{2+}$ concentrations in tiger shrimp hemolymph range from 6.4 to $10 \mathrm{mM}$ [28]. Thus, when TGH is released from hemocytes it would be fully activated to initiate the coagulation (Fig. 3). However, metal ions and high chloride ion [2] in the hemolymph possibly reduce its enzymatic activity within minutes, as a feedback regulation to avoid excess clotting.

The X-ray crystallographic structure $[33,34]$ showed that the catalytic domain (i.e., region 200-550) of FXIIIa contains a hydrogen-bonded Cys-His-Asp triad similar to that found in enzymes of the papain family [35]. The catalytic triad appears to be also conserved in PmTG (and Mj-TGH), i.e., Cys330 (325), His397 (392), Asp420 (415). Their $\mathrm{Ca}^{2+}$-binding sites presumably include the conserved Asn460, Asp462, Glu512, and Glu517 (Fig. 8). With highly conserved region 350-353, His 358 (353) and Glu451 (466) in shrimp TGH may be associated as in FXIIIa [33]. Many conserved Trp residues in the core domain of TGH (Fig. 8) possibly are involved in stabilization of the transition state during the enzymatic reaction [36]. It is known that $\mathrm{Ca}^{2+}$ unmasks the active site Cys and widens the protein substrate binding site $[1,37]$ and the active site possibly undergoes irreversible conformational changes. Being 35\% similar to FXIIIA, TGH is relatively unstable after activation at ambient temperature and in the presence of bivalent metal ions (Fig. 5). Instability of other transglutaminases has been reported before $[2,38]$, but its exact mechanism remains to be explained by $3 \mathrm{D}$ structural analyses of both the active and the inactive enzymes or by mutagenesis study [39].

The RGD (or Arg-Gly-Asp) motif, which is also present in fibrinogen, is known to bind to integrins or specific membrane receptors [40]. Positions of RGD motifs are remarkably conserved in all invertebrate transglutaminases (Fig. 8) and human keratinocyte [8]. This motif probably is essential for the shrimp enzyme to bind integrins and form complex with adhesion molecules $[1,41]$, and possibly trigger certain signal pathways, e.g., RhoA activation and cell spreading [42]. Interestingly, the RGD are all flanked by Pro and/or Gly residues (i.e., "proline brackets") and structurally constrained on the protein surface [43], consistent with their role for protein-protein interaction.

Both STG I and PmTG were transcribed in P. monodon hemocytes. However, results of the peptide mass fingerprinting of $P$. monodon TGH confirmed its identity with STG I (Table 1) but not with PmTG or STG II. The molecular mass of $P$. monodon TGH was determined as $84280 \pm 168 \mathrm{Da}$. The predicted P. monodon TGH mass would be $84713 \mathrm{Da}$ (based on the STG I sequence, Fig. 8) assuming no disulfide bond was formed and N-terminal acetylation took place after removal of Met 1, as in the case of FXIIIa [44]. Alternatively, the mass would be $84285 \mathrm{Da}$ if four residues (Met-Pro-Thr-Val) were removed. Thus, the determined mass suggests that TGH possibly is processed by deleting $\mathrm{N}$-terminal residues before acetylation but not glycosylated although they contain seven potential Nglycosylation sites (Figs. 6 and 7).

Differed by merely six amino acid substitutions, PmTG and STG II probably are alleles of the same locus with natural polymorphism. The phylogenetic tree (Fig. 9) shows that the shrimps have at least two paralogous transglutaminases, i.e., PmTG/STG II and STG I in P. monodon, and Mj-transglutaminase (AB162767) and Mj-TGH in M. japonica. Paralogs are homologous proteins resulted from gene-duplication and they usually co-expressed in the same organism, while orthologs are those from the same gene lineage but expressed in different organisms [45]. Previous immunodiffusion experiments showed that antibodies prepared against of $P$. monodon clottable protein reacted with M.japonicus clottable protein [32]. The coagulation systems for this two genetically related species [46] presumably are similar. Results in Fig. 8 confirmed a high structural similarity between the hemocyte enzymes (STG I and Mj-TGH) of both species, but many distinct substitutions between STG I and PmTG at their core regions (residues 200-500) suggesting possible different substrate specificities. Protein sequence of a previously cloned crayfish transglutaminase [9] appears to be more close to that of PmTG or STG II (62\%) than that of STG I $(55 \%)$, whether it is the only isozyme or the real functional TGH of this fresh-water Crustacean remains to be studied.

The recombinant STG I from insect-baculovirus expression system did not show clotting activities [8], and recombinant STG II was postulated to be the clotting enzyme of P. monodon [10]. However, STG I was expressed in Sf21 cells cultured at $26{ }^{\circ} \mathrm{C}$ and the cell lysate were frozen before assay, the recombinant STG I probably had lost most of its activity under the culture conditions or freeze-and-thaw procedure. The instability of STG I probably lead to the wrong conclusion that STG 1 is not responsible for the hemolymph coagulation [8]. Moreover, in vitro assays of recombinant STG II may also be misleading, since the clot generated by recombinant STG II was less branched and instable [10], not like the clot formed naturally in crayfish [47] and horseshoe crab [2].

Based on microscopic observation, shrimp hemocytes comprised of hyaline cells, semi-granular cells, and granular cells [48-50]. Coagulation in shrimp is mediated by the circulating hyaline cells [31]. We have cloned more cDNAs encoding PmTG than those encoding STG I although STG I was the only transglutaminase purified from P. monodon hemocytes. By RTPCR, we also found PmTG gene highly expressed in hemocytes and muscle, but less expressed in other tissues (Fig. 10), consistent with the expression patterns of STG II gene [10]. Thus, PmTG mRNA is relatively abundant in the hemocytes, while STG I gene is constitutive expressed at low-level in various tissues [8]. The mRNA of a crayfish transglutaminase (possibly orthologous to those of PmTG/STG II) was also reported to be expressed in semigranular and granular cells [9].

Since shrimp have an open circulatory system and hemocytes are likely to rest onto different tissue surfaces, the Northern blot 
results of PmTG (Fig. 10) need to be confirmed by in situ hybridization. Previous in situ hybridization pointed out a low mRNA level of STG I in circulating hemocytes but a high level in mitotic cells of hematopoietic tissue [8], and the RT-PCR experiment also showed that STG I mRNA is decreased when hemocytes matured and released into the hemolymph. Taken together, these results suggest that transcription and translation of STG I in the hemocytes possibly take place in early developmental stages but down-regulated in later developmental stages.

In conclusion, this is the first biochemical study on shrimp hemocyte transglutaminases (TGH) responsible for hemolymph coagulation. The purified shrimp TGHs are shown to be relatively unstable after $\mathrm{Ca}^{2+}$-dependent activation. We also cloned two novel transglutaminases, PmTG from $P$. monodon and Mj-TGH from M. japonicus, and confirmed the presence of two transglutaminase genes in both species. By peptide mass fingerprinting (Table 1) or sequencing of purified TGH peptide fragments (Fig. 8), both STG I and Mj-TGH have been identified to be the functional TGH in the two shrimp species. We failed to isolate their paralogous enzymes, PmTG/STG II and another transglutaminase previously cloned from M.japonicus, although their mRNAs were expressed in shrimp hemocytes. The specific role and regulated expression of these transglutaminases in different Crustacean remain to be investigated.

\section{Acknowledgements}

We thank Yuh-Ling Chen for supplying peptide sequence data of M. japonica transglutaminase and Chien-Hong Lu for preparing the shrimp cDNA. MALDI-TOF/TOF analyses were carried out by the Core Facilities for Proteomics and Structural Biology Research, Institute Biological Chemistry, Academia Sinica, Taiwan. This research was supported by grants from Academia Sinica and Hung Kuang University, Taiwan, ROC.

\section{References}

[1] L. Lorand, R.M. Graham, Transglutaminases, cross-linking enzymes with pleiotropic functions, Nat. Rev., Mol. Cell Biol. 4 (2003) 140-156.

[2] F. Tokunaga, M. Yamada, T. Miyata, Y.L. Ding, M. Hiranaga-Kawabata, T. Muta, S. Iwanaga, A. Ichinose, E.W. Davie, Limulus hemocyte transglutaminase cDNA cloning, amino acid sequence, and tissue localization, J. Biol. Chem. 268 (1993) 252-261.

[3] C.S. Greenberg, P.J. Brickbichler, R.H. Rice, Transglutaminases: multifunction cross-linking enzymes that stabilize tissue, FASEB J. 5 (1991) 3071-3077.

[4] D. Aeschlimann, M. Paulsson, Transglutaminase: protein crosslinking enzymes in tissues and body fluid, Thromb. Haemost. 71 (1994) 402-415.

[5] V.C. Yee, I. Le Trong, P.D. Bishop, L.C. Pedersen, R.E. Stenkamp, D.C. Teller, Structure and function studies of factor XIIIa by X-ray crystallography, Semin. Thromb. Hemost. 22 (1996) 377-384.

[6] E. Candi, G. Melino, G. Mei, E. Tarcsa, S.I. Chung, L.N. Marekov, P.M. Steinert, Biochemical, structural, and transglutaminase substrate properties of human lorcrin, the major epidermal cornified cell envelope protein, J. Biol. Chem. 270 (1995) 26382-26390.

[7] H.J. Lin, C.W. Lo, Y.H. Chen, Localization of the transglutaminase crosslinking site in SVS III, a novel glycoprotein secreted from mouse seminal vesicle, J. Biol. Chem. 277 (2002) 3632-3639.

[8] C.C. Huang, K. Sritunyalucksana, K. Soderhall, Y.L. Song, Molecular cloning and characterization of tiger shrimp (Penaeus monodon) transglutaminase, Dev. Comp. Immunol. 28 (2004) 279-294.
[9] R. Wang, Z. Liang, M. Hall, K. Soderhall, A transglutaminase involved in the coagulation system of the freshwater crayfish. Pacifastacus leniusculus. Tissue localization and cDNA cloning, Fish Shellfish Immunol. 11 (2001) 623-637.

[10] M.Y. Chen, K.Y. Hu, C.C. Huang, Y.L. Song, More than one type of transglutaminase in invertebrates? A second type of transglutaminase is involved in shrimp coagulation, Dev. Comp. Immunol. 29 (2005) $1003-1016$.

[11] F. Tokunaga, T. Muta, S. Iwanaga, A. Ichinose, E.W. Davie, K. Kuma, T. Miyata, Limulus hemocyte transglutaminase cDNA cloning, amino acid sequence, and tissue localization, J. Biol. Chem. 268 (1993) 262-268.

[12] P.M. Steinert, S.Y. Kim, S.I. Chung, L.N. Marekov, The transglutaminase enzyme is variably acylated by myristate and palmitate during differentiation in epidermal keratinocytes, J. Biol. Chem. 271 (1996) 26242-26250.

[13] G. Melino, M. Piacentini, Tissue transglutaminase in cell death: a downstream or a multifunctional upstream effector, FEBS Lett. 430 (1998) 59-63.

[14] M.S. Yeh, C.J. Huang, J.H. Leu, Y.C. Lee, I.H. Tsai, Molecular cloning and characterization of a hemolymph clottable protein from tiger shrimp (Penaeus monodon), Eur. J. Biochem. 266 (1999) 624-633.

[15] L. Lorand, O.M. Lockridge, L.K. Campbell, R. Myhrman, J. BrunerLorand, Transamidating enzymes II. A continuous fluorescent method suited for automating measurements of factor XIII in plasma, Anal. Biochem. 4 (1971) 221-231.

[16] S.C. Brenner, F. Wold, Human erythrocyte transglutaminase purification and properties, Biochim. Biophys. Acta 522 (1978) 74-84.

[17] U.K. Laemmli, Cleavage of structural proteins during the assembly of the head of bacteriophage T4, Nature 227 (1970) 680-685.

[18] J. Sambrook, E.F. Fritsch, T. Maniatis, Molecular Cloning: A Laboratory Manual, Cold Spring Harbor Laboratory Press, Cold Spring Harbor, New York, 1989

[19] P. Chomczynski, N. Sacchi, Single-step method of RNA isolation by acid guanidinium thiocyanate-phenol-chloroform extraction, Anal. Biochem. 162 (1987) 156-159

[20] K.B. Mullis, F.A. Faloona, Specific synthesis of DNA in vitro via a polymerase-catalyzed chain reaction, Methods Enzymol. 155 (1987) 335-350.

[21] F. Sanger, S. Nicklen, A.R. Coulson, DNA sequencing with chainterminating inhibitors, Proc. Natl. Acad. Sci. U. S. A. 74 (1977) 5463-5467.

[22] S. McGinnis, T.L. Madden, BLAST: at the core of a powerful and diverse set of sequence analysis tools, Nucleic Acids Res. 32 (2004) W20-W25.

[23] J.D. Thompson, D.G. Higgins, T.J. Gibson, CLUSTAL W: improving the sensitivity of progressive multiple sequence alignment through sequence weighting, position-specific gap penalties and weight matrix choice, Nucleic Acids Res. 22 (1994) 4673-4680.

[24] J. Felsenstein, PHYLIP: the PHYLogeny Inference Package, version 3.573. Computer program distributed by the U. of Washington, Dept. of Genetics, Seattle, 1992.

[25] J. Felsenstein, Confidence limits on phylogenies: an approach using the bootstrap, Evolution 39 (1985) 783-791.

[26] C.G. Curtis, K.L. Brown, R.B. Credo, R.A. Domanik, A. Gray, P. Stenberg, L. Lorand, Calcium-dependent unmasking of active center cysteine during activation of fibrin stabilizing factor, Biochemistry 13 (1974) 3774-3780.

[27] R.B. Credo, C.G. Curtis, L. Lorand, Alpha-chain domain of fibrinogen controls generation of fibrinoligase (coagulation factor XIIIa). Calcium ion regulatory aspects, Biochemistry 20 (1981) 3770-3778.

[28] R.P. Ferraris, Fe D. Parado-Estepa, J.M. Ladja, Effect of salinity on the osmotic, chloride, total protein and calcium concentrations in the hemolymph of the prawn Penaeus monodon (Fabricius), Comp. Biochem. Physiol. 83A (1986) 701-708.

[29] M. Kozak, An analysis of 50-noncoding sequences from 699 vertebrate messenger RNAs, Nucleic Acids Res. 15 (1987) 8125-8148.

[30] P. Kopacek, M. Hall, K. Sorderhall, Characterization of a clotting protein isolated from the plasma of the freshwater crayfish Pacifastcaus leniusculus, Eur. J. Biochem. 213 (1993) 591-597.

[31] S.A. Omori, G.G. Martin, J.E. Hose, Morphology of hemocyte lysis and clotting in the ridge back prawn, Sicyonia ingentis, Cell Tissue Res. 255 (1989) $117-123$. 
[32] M.S. Yeh, Y.L. Chen, I.H. Tsai, The hemolymph clottable proteins of tiger shrimp, Penaeus monodon, and related species, Comp. Biochem. Physiol. 121B (1998) 169-176.

[33] V.C. Yee, L.C. Pedersen, I. Le Trong, P.D. Bishop, R.E. Stenkamp, D.C. Teller, Three-dimensional structure of a transglutaminase: human blood coagulation factor XIIIa, Proc. Natl. Acad. Sci. U. S. A. 91 (1994) 7296-7300.

[34] B.A. Fox, V.C. Yee, L.C. Pedersen, I. Le Trong, P.D. Bishop, R.E. Stenkamp, D.C. Teller, Identification of the calcium binding site and a novel ytterbium site in blood coagulation factor XIII by X-ray crystallography, J. Biol. Chem. 274 (1999) 4917-4923.

[35] L.C. Pedersen, V.C. Yee, P.D. Bishop, I. Le Trong, D.C. Teller, R.E. Stenkamp, Transglutaminase factor XIII uses protease-like catalytic triad to crosslink macromolecules, Protein Sci. 3 (1994) 1131-1135.

[36] S.E. Iismaa, S. Holman, M.A. Wouters, L. Lorand, R.M. Graham, A. Husain, Evolutionary specialization of a tryptophane indole group for transition-state stabilization by eukaryotic transglutaminases, Proc. Natl. Acad. Sci. U. S. A. 100 (2003) 12636-12641.

[37] R. Casadio, E. Polverini, P. Mariani, F. Spinozzi, F. Carsughi, A. Fontana, P. Polverino de Laureto, G. Matteucci, C.M. Bergamini, The structural basis for the regulation of tissue transglutaminase by calcium ions, Eur. J. Biochem. 262 (1999) 672-679.

[38] J.E. Folk, P.W. Cole, Transglutaminase: mechanistic features of the active site as determined by kinetic and inhibitor studies, Biochim. Biophys. Acta 122 (1966) 244-264.

[39] V. Schroeder, E. Meili, T. Cung, P. Schmutz, H.P. Kohler, Characterisation of six novel A-subunit mutations leading to congenital factor XIII deficiency and molecular analysis of the first diagnosed patient with this rare bleeding disorder, Thromb. Haemost. 95 (2006) 77-84.

[40] E. Ruoslahti, RGD and other recognition sequences for integrins, Annu. Rev. Cell Dev. Biol. 12 (1996) 697-715.
[41] S.S. Akimov, A.M. Belkin, Cell surface tissue transglutaminase is involved in adhesion and migration of monocytic cells on fibronectin, Blood 98 (2001) 1567-1576.

[42] A. Salsmann, E. Schaffner-Reckinger, F. Kabile, S. Plancon, N. Kieffer, A new functional role of the fibrinogen RGD motif as the molecular switch that selectively triggers integrin alphaIIbbeta3-dependent RhoA activation during cell spreading, J. Biol. Chem. 280 (2005) 33610-33619.

[43] R.M. Kini, Proline brackets and identification of potential functional sites in proteins: toxins to therapeutics, Toxicon 36 (1998) 1659-1670.

[44] K. Ikura, H. Yokota, R. Sasaki, H. Chiba, Determination of amino- and carboxyl-terminal sequences of guinea pig liver transglutaminase: evidence for amino terminal processing, Biochemistry 28 (1989) 2344-2348.

[45] M. Kanehisa, S. Goto, S. Kawashima, A. Nakaya, The KEGG databases at GenomeNet, Nucleic Acids Res. 30 (2002) 42-46.

[46] S. Lavery, T.Y. Chan, Y.K. Tam, K.H. Chu, Phylogenetic relationships and evolutionary history of the shrimp genus Penaeus s.l. derived from mitochondrial DNA, Mol. Phylogenet. Evol. 31 (2004) 39-49.

[47] M. Hall, R. Wang, R. van Antwerpen, L. Sottrup-Jensen, K. Soderhall, The crayfish plasma clotting protein: a vitellogenin-related protein responsible for clot formation in crustacean blood, Proc. Natl. Acad. Sci. U. S. A. 96 (1999) 1965-1970.

[48] A. Tsing, J.M. Arcier, M. Brehelin, Hemocytes of Penaeid and Palaemonid shrimps: morphology, cytochemistry, and hemograms, J. Invertebr. Pathol. 53 (1989) 64-77.

[49] J. Rodriguez, V. Boulov, E. Mialhe, E. Bachere, Characterization of shrimp haemocytes and plasma components by monoclonal antibodies, J. Cell Sci. 108 (1995) 1043-1050.

[50] C.B.T. Van de Braak, R. Faber, J.H. Boon, Cellular and humoral characteristics of Penaeus monodon (Fabricius, 1798) haemolyph, Comp. Haematol. Int. 6 (1996) 194-203. 